M. Itô and N. Suzuki

Nagoya Math. J.

Vol. 83 (1981), 53-106

\title{
COMPLETELY SUPERHARMONIC MEASURES FOR THE INFINITESIMAL GENERATOR $A$ OF A DIFFUSION SEMI-GROUP AND POSITIVE EIGEN ELEMENTS OF $A$
}

\author{
MASAYUKI ITÔ AND NORIAKI SUZUKI
}

\section{§1. Introduction}

Let $X$ be a locally compact Hausdorff space with countable basis. We denote by

$M(X)$ the topological vector space of all real Radon measures in $X$ with the vague topology,

$M_{K}(X)$ the topological vector space of all real Radon measures in $X$ whose supports are compact with the usual inductive limit topology.

Their subsets of all non-negative Radon measures are denoted by $M^{+}(X)$ and by $M_{K}^{+}(X)$, respectively.

In the paragraph 2, we shall prepare the terminology and the notation which we shall use in the sequel.

A continuous linear operator $T$ from $M_{K}(X)$ into $M(X)$ is called a diffusion kernel on $X$ if $T$ is positive, i.e., $T \mu \in M^{+}(X)$ whenever $\mu \in M_{K}^{+}(X)$. A semi-group $\left(T_{t}\right)_{t \geq 0}$ of diffusion kernels on $X$ is called a diffusion semigroup if $T_{0}=I$ (the identity) and if, for any $\mu \in M_{K}(X)$, the mapping $t \rightarrow$ $T_{t} \mu$ is continuous in $M(X)$.

We consider the infinitesimal generator $A$ of a transient and regular diffusion semi-group $\left(T_{t}\right)_{t \geq 0}$ on $X$. A Radon measure $\mu \in M(X)$ is said to be $A$-superharmonic (resp. $A$-harmonic) if it satisfies $-A \mu \in M^{+}(X)$ (resp. $A \mu=0)$.

In the paragraph 3 , we shall show that every positive $A$-superharmonic Radon measure is written uniquely as the sum of a $V$-potential of a nonnegative Radon measure and a non-negative $A$-harmonic measure, where $V$ is the Hunt diffusion kernel for $\left(T_{t}\right)_{t \geq 0}$, i.e.,

Received April 27, 1979. 


$$
V=\int_{0}^{\infty} T_{t} d t
$$

By generalizing the classical positive eigen equation with zero conditions on the boundary and by defining that a Radon measure vanishes $V$-n.e. on the boundary (Definition 21 in $\$ 2$ ), we shall discuss, in the paragraph 4, a positive eigen equation for $A$ with zero conditions in the following setting:

For a positive number $c>0$,

$$
\left\{\begin{aligned}
A \mu & =-c \mu \\
\mu & =0 V \text {-n.e. on the boundary. }
\end{aligned}\right.
$$

Denote by $E_{0}(A ; c)$ the set of all non-negative solutions of (1.2) and put $E_{0}(A)=\bigcup_{c \geqq 0} E_{0}(A ; c)$. Under the assumption that $A$ satisfies the condition $(\mathscr{L})$ (Definition 49 in $\S 4$ ), we shall show that $E_{0}(A)$ is a Borel measurable set in the metrizable space $M^{+}(X)$.

By generalizing the notion of the classical complete superharmonicity, we define the complete $A$-superharmonicity of $\mu \in M(X)$. A Radon measure $\mu \in M(X)$ is said to be completely $A$-superharmonic if, for any integer $n \geqq 1,(-A)^{n} \mu \in M^{+}(X)$, where $(-A)^{n}$ denotes the $n$-th iterate of $-A$. Let $S C(A)$ be the set of all non-negative completely $A$-superharmonic measures in $X$ and put

$$
\begin{aligned}
& S C_{0}(A)=\left\{\mu \in S C(A) ;(-A)^{n} \mu=0 V\right. \text {-n.e. on the boundary } \\
& \text { for } n=0,1, \cdots\} .
\end{aligned}
$$

Under the condition $(\mathscr{L})$ for $A, S C(A)$ is a closed convex cone in $M^{+}(X)$ and all extreme rays of $S C(A)$ contained in $S C(A)-S C_{0}(A)$ are determined whenever all extreme rays of $S C(A)$ contained in $H(A)$ are determined, where $H(A)$ is the convex cone formed by all non-negative $A$-harmonic measures.

A main purpose of the paragraph 4 is to show that

$$
\begin{aligned}
S C_{0}(A) & =\left\{\int \nu d \Phi(\nu) \in M^{+}(X) ; \Phi \in M_{b}^{+}\left(E_{0}(A)\right)\right\} \\
& =\left\{\int_{0}^{\infty} \mu_{t} d \sigma(t) \in M^{+}(X) ; \mu_{t} \in E_{0}(A ; t), \sigma \in M_{b}^{+}((0, \infty))\right\},
\end{aligned}
$$

where $M_{b}^{+}\left(E_{0}(A)\right)$ and $M_{b}^{+}((0, \infty))$ denote the set of all regular Borel nonnegative measures $\Phi$ on $E_{0}(A)$ with $\int d \Phi<\infty$ and that of all Borel non- 
negative measures $\sigma$ in $(0, \infty)$ with $\int d \sigma<\infty$, respectively. Let $A=d / d x$ in $(0, \infty)$. Then (1.4) implies the Bernstein theorem.

M. V. Noviskiî [16] discussed a similar formula as in (1.4) for the infinitesimal generator of a contraction semi-group in a Banach space.

In the paragraph 5 , for a given elliptic differential operator $L$ of second order on a subdomain $D$ of an orientable $C^{\infty}$-manifold, we shall show that the diffusion semi-group defined by the fundamental solution of $\partial / \partial t-L$ is regular if it is transient. Applying our theorem to completely $L$-superharmonic functions in $D$, we shall obtain the integral representation of a completely $L$-superharmonic function in $D$. This is a generalization of Noviskiř's result (see [15]).

\section{§2. Basic notation and preliminaries}

We denote by

$C(X)$ the Fréchet space of all real-valued continuous functions in $X$ with the topology of compact uniform convergence,

$C_{K}(X)$ the topological vector space of all real-valued continuous functions in $X$ whose supports are compact with the usual inductive limit topology.

Their subsets of all non-negative functions are also denoted by $C^{+}(X)$ and $C_{K}^{+}(X)$, respectively.

Definition 1. (1) A continuous linear operator $T$ from $M_{K}(X)$ into $M(X)$ is called a diffusion kernel if $T$ is positive, i.e., $T \mu \in M^{+}(X)$ whenever $\mu \in M_{K}^{+}(X)$.

(2) A linear operator $T$ from $C_{K}(X)$ into $C(X)$ is called a continuous kernel if $T$ is positive, i.e., $T f \in C^{+}(X)$ whenever $f \in C_{K}^{+}(X)$.

Remark 2. A continuous kernel $T$ is a continuous mapping from $C_{K}(X)$ into $C(X)$.

We see easily the following

Remark 3. (1) Let $T$ be a diffusion kernel on $X$. For $f \in C_{K}(X)$, we put

$$
T^{*} f(x)=\int f d T \varepsilon_{x},
$$

where $\varepsilon_{x}$ denotes the Dirac measure at $x \in X$. Then $T^{*} f \in C(X)$ and $T^{*}: C_{K}(X) \ni f \rightarrow T^{*} f \in C(X)$ is a continuous kernel on $X$. 
(2) Let $T$ be a continuous kernel on $X$. For $\mu \in M_{K}(X)$, there exists one and only one $T^{*} \mu \in M(X)$ such that, for any $f \in C_{K}(X)$,

$$
\int f d T^{*} \mu=\int T f d \mu
$$

and $T^{*}: M_{K}(X) \ni \mu \rightarrow T^{*} \mu \in M(X)$ is a diffusion kernel on $X$.

In (1), $T^{*}$ is called the dual continuous kernel of $T$ and in (2), $T^{*}$ is the dual diffusion kernel of $T$.

Remark 4. Let $T$ be a diffusion kernel or a continuous kernel on $X$. Then $\left(T^{*}\right)^{*}=T$.

In the sequel, for a diffusion kernel or a continuous kernel $T$, its dual kernel is always denoted by $T^{*}$. For a diffusion kernel $T$ on $X$, we put

$$
\mathscr{D}(T)=\left\{\mu \in M(X) ; \int T^{*} f d|\mu|<\infty \text { for all } f \in C_{K}^{+}(X)\right\},
$$

where $|\mu|$ denotes the total variation of $\mu$, and put $\mathscr{D}^{+}(T)=\mathscr{D}(T) \cap M^{+}(X)$. Then $\mathscr{D}(T)$ is a linear subspace of $M(X)$ and $T$ can be extended to a positive linear operator from $\mathscr{D}(T)$ into $M(X)$. For $\mu \in \mathscr{D}(T), T \mu$ is called the $T$-potential of $\mu$.

Let $T$ be a continuous kernel on $X$. Put

$$
\begin{array}{r}
\mathscr{D}(T)=\left\{f \in C(X) ; \int|f| d T^{*} \mu<\infty \text { for all } \mu \in M_{K}^{+}(X)\right. \text { and } \\
\left.M_{K}(X) \ni \mu \rightarrow \int f d T^{*} \mu \text { is continuous }\right\} .
\end{array}
$$

Then, by the following lemma and Remark 4, we see that $\mathscr{D}(T)$ is a linear subspace of $C(X)$ and that $T$ can be extended to a positive linear operator from $\mathscr{D}(T)$ into $C(X)$ by defining $T f(x)=\int f d T^{*} \varepsilon_{x}$.

LEMma 5. Let $T$ and $\mathscr{D}(T)$ be the same as above. If $f \in C(X)$ and $|f| \leqq|g|$ for some $g \in \mathscr{D}(T)$, then $f \in \mathscr{D}(T)$.

In fact, Lemma 5 follows from the lower semi-continuity of the function $\int h d T^{*} \varepsilon_{x}$ of $x$ for all $h \in C^{+}(X)$.

Let $T_{j}(j=1,2)$ be a diffusion kernel (resp. a continuous kernel) on $X$. If, for any $\mu \in M_{K}(X)$ (resp. $f \in C_{K}(X)$ ), $T_{2} \mu \in \mathscr{D}\left(T_{1}\right)$ (resp. $T_{2} f \in \mathscr{D}\left(T_{1}\right)$ ) and if the mapping $\mu \rightarrow T_{1}\left(T_{2} \mu\right)$ (resp. $f \rightarrow T_{1}\left(T_{2} f\right)$ ) defines a diffusion kernel (resp. a continuous kernel), it is called the product of $T_{1}$ and $T_{2}$ and 
denoted by $T_{1} \cdot T_{2}$.

Remark 6. Let $T_{j}(j=1,2)$ be a diffusion kernel (resp. a continuous kernel) on $X$. If $T_{1} \cdot T_{2}$ is defined, then $T_{2}^{*} \cdot T_{1}^{*}$ is defined and $\left(T_{1} \cdot T_{2}\right)^{*}=$ $T_{2}^{*} \cdot T_{1}^{*}$.

In particular, for a diffusion kernel $T$ (resp. a continuous kernel) on $X$ and a positive integer $n \geqq 2$, we denote by $T^{n}$ the diffusion kernel (resp. the continuous kernel) defined inductively by $T^{n-1} \cdot T$ provided that it is defined, where $T^{1}=T$. In the case of $T \neq 0, T^{0}$ means the identity $I$.

Definition 7. A family $\left(T_{t}\right)_{t \geqq 0}$ of diffusion kernels (resp. continuous kernels) on $X$ is called a diffusion semi-group (resp. continuous semi-group) if it satisfies the following three conditions:

$$
\begin{gathered}
T_{0}=I . \\
T_{t} \cdot T_{s}=T_{t+s} \text { for any } t \geqq 0, s \geqq 0 .
\end{gathered}
$$

For each $\mu \in M_{K}(X)$ (resp. $f \in C_{K}(X)$ ), the mapping $t \rightarrow T_{t} \mu$ (resp. $\left.t \rightarrow \int T_{t} f d \mu\right)$ is continuous in $M(X)$ (resp. continuous for each $\left.\mu \in M_{K}(X)\right)$.

Evidently, for a diffusion semi-group (resp. a continuous semi-group) $\left(T_{t}\right)_{t \geqq 0},\left(T_{t}^{*}\right)_{t \geqq 0}$ is a continuous semi-group (resp. a diffusion semi-group).

Let $\left(T_{t}\right)_{t \geqq 0}$ be a diffusion semi-group (resp. a continuous semi-group) on $X$. Putting

$$
\begin{array}{r}
\mathscr{D}\left(\left(T_{t}\right)_{t \geqq 0}\right)=\left\{\mu \in \bigcap_{t \geqq 0} \mathscr{D}\left(T_{t}\right) ; t \longrightarrow T_{t} \mu \text { is continuous in } M(X)\right\} \\
\text { (resp. } \mathscr{D}\left(\left(T_{t}\right)_{t \geqq 0}\right)=\left\{f \in \bigcap_{t \geqq 0} \mathscr{D}\left(T_{t}\right) ; t \longrightarrow \int T_{t} f d \mu\right. \text { is continuous for } \\
\text { each } \left.\left.\mu \in M_{K}(X)\right\}\right),
\end{array}
$$

we call it the domain of $\left(T_{t}\right)_{t \geqq 0}$. We put also $\mathscr{D}^{+}\left(\left(T_{t}\right)_{t \geqq 0}\right)=\mathscr{D}\left(\left(T_{t}\right)_{t \geqq 0}\right) \cap$ $M^{+}(X)$ (resp. $\left.=\mathscr{D}\left(\left(T_{t}\right)_{t \geqq 0}\right) \cap C^{+}(X)\right)$.

Definition 8. Let $\left(T_{t}\right)_{t \geqq 0}$ be a diffusion semi-group (resp. a continuous semi-group) on $X$. We say that it is transient if the mapping $V: M_{K}(X) \ni \mu$ $\rightarrow \int_{0}^{\infty} T_{t} \mu d t \in M(X)\left(\right.$ resp. $\left.C_{K}(X) \ni f \rightarrow \int_{0}^{\infty} T_{t} f d t \in C(X)\right)$ is defined as a diffu- 
sion kernel (resp. a continuous kernel) on $X$, where, for any $f \in C_{K}(X)$, $\int f d\left(\int_{0}^{\infty} T_{t} \mu d t\right)=\int_{0}^{\infty} \int f d T_{t} \mu d t$

In this case, we denote by

$$
V=\int_{0}^{\infty} T_{t} d t
$$

and call it the Hunt diffusion kernel for $\left(T_{t}\right)_{t \geqq 0}$ (resp. the Hunt continuous kernel for $\left.\left(T_{t}\right)_{t \geqq 0}\right)$.

Evidently we see the following

Remark 9. Let $\left(T_{t}\right)_{t \geq 0}$ be a diffusion semi-group (resp. a continuous semi-group) on $X$. Then $\left(T_{t}\right)_{t \geqq 0}$ is transient if and only if $\left(T_{t}^{*}\right)_{t \geq 0}$ is transient.

Furthermore, in the case that $\left(T_{t}\right)_{t \geqq 0}$ is transient, we have

$$
\left(\int_{0}^{\infty} T_{t} d t\right)^{*}=\int_{0}^{\infty} T_{t}^{*} d t
$$

Let $\left(T_{t}\right)_{t \geqq 0}$ be a transient diffusion semi-group (resp. a transient continuous semi-group) on $X$. For any $p \geqq 0$, we put

$$
V_{p}=\int_{0}^{\infty} \exp (-p t) T_{t} d t
$$

and call $\left(V_{p}\right)_{p \geqq 0}$ the resolvent for $\left(T_{t}\right)_{t \geqq 0}$. In this case, $V_{p}$ is a diffusion kernel (resp. a continuous kernel, because the Fatou lemma gives that, for any $f \in C_{K}^{+}(X), V_{p} f$ and $V f-V_{p} f$ are lower semi-continuous).

In the usual way, we see the following

Proposition 10. (1) Let $\left(T_{t}\right)_{t \geqq 0}$ and $\left(T_{t}^{\prime}\right)_{t \geqq 0}$ be transient diffusion semigroups (resp. transient continuous semi-groups) on $X$. If $\int_{0}^{\infty} T_{t} d t=\int_{0}^{\infty} T_{t}^{\prime} d t$, then $T_{t}=T_{t}^{\prime}$ for any $t \geqq 0$.

(2) Let $\left(T_{t}\right)_{t \geqq t}$ be the same as above and $V$ be the Hunt diffusion kernel (resp. the Hunt continuous kernel) for $\left(T_{t}\right)_{t \geqq 0}$. If a family $\left(V_{p}\right)_{p \geqq 0}$ of diffusion kernels (resp. continuous kernels) satisfies the following

$$
\begin{aligned}
& V_{p}-V_{q}=(q-p) V_{p} \cdot V_{q} \text { for any } p \geqq 0 \text { and } q>0, \text { and } \\
& \lim _{p \rightarrow 0} V_{p}=V_{0}=V,
\end{aligned}
$$

then $\left(V_{p}\right)_{p \geqq 0}$ is the resolvent for $\left(T_{t}\right)_{t \geqq 0}$. 
We remark here that $\lim _{p \rightarrow 0} V_{p}=V_{0}$ means that, for any $\mu \in M_{K}(X)$, $\lim _{p \rightarrow 0} V_{p} \mu=V_{0} \mu$ in $M(X)$ (resp. for any $f \in C_{K}(X), \lim _{p \rightarrow 0} V_{p} f=V_{0} f$ in $C(X))$. For a transient continuous semi-group $\left(T_{t}\right)_{t \geqq 0}$, the Dini theorem gives that $\lim _{p \rightarrow 0} V_{p} f=V_{0} f$ in $C(X)$ if and only if $\lim _{p \rightarrow 0} V_{p} f(x)=V_{0} f(x)$ for each $x \in X$. The first equality in (2.12) is called the resolvent equation.

Proof of Proposition 10. We shall show only Proposition 10 for transient diffusion semi-groups, because the proof of the other case is similar. Let $\left(V_{1, p}\right)_{p \geq 0}$ and $\left(V_{2, p}\right)_{p \geq 0}$ be the resolvent for $\left(T_{t}\right)_{t \geq 0}$ and that for $\left(T_{t}^{\prime}\right)_{t \geq 0}$, respectively. Evidently we have $\lim _{p \rightarrow 0} V_{j, p}=V_{j, 0}(j=1,2)$. For each $p \geqq 0$, we put $H_{p}(t)=\exp (-p t)$ on $[0, \infty)$ and $=0$ in $(-\infty, 0)$. Then, for any $p \geqq 0$ and $q>0, H_{p}-H_{q}=(q-p) H_{p} * H_{q}$. By the Fubini theorem and (2.7), $\left(V_{j, p}\right)_{p \geqq 0}$ satisfies the resolvent equation. Since, for any $\mu \in M_{K}(X)$, the mappings $t \rightarrow T_{t} \mu$ and $t \rightarrow T_{t}^{\prime} \mu$ are continuous in $M(X)$, the above argument and the injectivity of the Laplace transformation show that (2) implies (1). We shall show (2). It suffices to show that, for any $p>0$ and any integer $n \geqq 1,\left(V_{p}\right)^{n}$ and $\left(V_{1, p}\right)^{n}$ are defined and

$$
V+\frac{1}{p} I=\frac{1}{p}\left(I+\sum_{n=1}^{\infty}\left(p V_{p}\right)^{n}\right)=\frac{1}{p}\left(I+\sum_{n=1}^{\infty}\left(p V_{1, p}\right)^{n}\right),
$$

where $\left(V_{1, p}\right)_{p \geqq 0}$ is the resolvent for $\left(T_{t}\right)_{t \geq 0}$, because $\left(I-p V_{p}\right) \cdot(p V+I)$. $\left(I-p V_{p}\right)=\left(I-p V_{p}\right) \cdot(p V+I) \cdot\left(I-p V_{1, p}\right)$. By using the resolvent equation, we see that $\left(V_{p}\right)^{n}$ and $\left(V_{1, p}\right)^{n}$ are defined $(n=1,2, \cdots)$. We shall show only the first equality in (2.13), because the other is similar. This follows directly from

$$
V_{q}+\frac{1}{p-q} I=\frac{1}{p-q}\left(I+\sum_{n=1}^{\infty}\left((p-q) V_{p}\right)^{n}\right)
$$

for any $q$ with $0<q<p$, because, for any $\mu \in M_{K}^{+}(X), V_{q} \mu \uparrow V \mu$ with $q \downarrow 0$. By the resolvent equation, we have

$$
\begin{aligned}
& \frac{1}{p-q}\left(I+\sum_{n=1}^{\infty}\left((p-q) V_{p}\right)^{n}\right) \\
& \quad=\frac{1}{p-q} I+V_{q}-\lim _{n \rightarrow \infty}\left(\frac{1}{p-q} I+V\right) \cdot\left((p-q) V_{p}\right)^{n} \\
& \quad=\frac{1}{p-q} I+V_{q},
\end{aligned}
$$

because, for any $\mu \in \mathscr{D}^{+}(V)$, 


$$
(p-q)^{n} V\left(V_{p}\right) \mu \leqq\left(\frac{p-q}{p}\right)^{n} V \mu
$$

This completes the proof.

Definition 11. A continuous kernel $V$ on $X$ is said to satisfy the domination principle if, for any $f, g \in C_{K}^{+}(X)$, an inequality $V f(x) \leqq V g(x)$ on the support of $f$, supp $(f)$, implies the same inequality on $X$.

Proposition 12. Let $\left(T_{t}\right)_{t \geqq 0}$ be a transient continuous semi-group and $V$ be the Hunt continuous kernel for $\left(T_{t}\right)_{t \geq 0}$. Then $V$ satisfies the domination principle.

If $X$ has a structure of an abelian group with which the topology of $X$ is compatible and if, for any $t \geqq 0, T_{t}$ is defined by a positive Radon measure $\alpha_{t}$ as follows;

$$
T_{t} f(x)=\alpha_{t} * f(x),
$$

then $\left(T_{t}\right)_{t \geqq 0}$ and $V$ are said to be of convolution type. The assertion of Proposition 12 is well-known in the case that $\left(T_{t}\right)_{t \geqq 0}$ is of convolution type (see, for example, [8]). Its proof is also valid in general case.

Proof of Proposition 12. Let $\left(V_{p}\right)_{p \geqq 0}$ be the resolvent for $\left(T_{t}\right)_{t \geqq 0}$ and suppose that, for $f, g \in C_{K}^{+}(X), V f(x) \leqq V g(x)$ on $\operatorname{supp}(f)$. Let $h \in C_{K}^{+}(X)$ such that $h(x)>0$ on $\operatorname{supp}(f)$. Then, for any $x_{0} \in \operatorname{supp}(f)$, there exists $t_{0}>0$ such that $T_{t} h\left(x_{0}\right)>0$ for all $t$ with $0<t<t_{0}$. Hence $V h\left(x_{0}\right)>0$, i.e., $V h(x)>0$ on $\operatorname{supp}(f)$. For any integer $n \geqq 1$, there exists $p_{0}>0$ such that, for any $p>p_{0}$,

$$
\left(V+\frac{1}{p} I\right) f(x) \leqq\left(V+\frac{1}{p} I\right)\left(g+\frac{1}{n} h\right)(x) \text { on } \operatorname{supp}(f) .
$$

Put $u=\inf ((V+(1 / p) I) f,(V+(1 / p) I)(g+(1 / n) h))$. Then we have

$$
\left(I-p V_{p}\right)\left(\left(V+\frac{1}{p} I\right) f-u\right)=p V_{p}\left(u-\left(V+\frac{1}{p} I\right) f\right) \leqq 0
$$

$$
\text { on } \operatorname{supp}(f) \text {. }
$$

Since $\left(I-p V_{p}\right)(V+(1 / p) I) f=(1 / p) f$ and $\left(I-p V_{p}\right) u \geqq 0$ on $X$, we have $\left(I-p V_{p}\right)((V+(1 / p) I) f-u) \leqq 0$, which gives that $(V+(1 / p) I) f \leqq u$ on $X$, i.e., $u=(V+(1 / p) I) f$ on $X$. Hence the inequality in (2.18) holds on $X$. Letting $p \rightarrow \infty$ and $n \rightarrow \infty$, we obtain that $V f(x) \leqq V g(x)$ on $X$. Thus Proposition 12 is shown. 
Remark 13. Let $V$ be the same as above. If, for $f, g \in \mathscr{D}^{+}(V), V f \leqq V g$ on $\operatorname{supp}(f)$, then the same inequality holds on $X$.

In fact, for any $f^{\prime} \in C_{K}^{+}(X)$ with $f^{\prime} \leqq f$, there exists $h \in C_{K}^{+}(X)$ such that $V h(x)>0$ on $\operatorname{supp}\left(f^{\prime}\right)$. Hence, for any integer $n \geqq 1$, there exists $g_{n} \in C_{K}^{+}(X)$ such that $g_{n} \leqq g$ and $V f^{\prime} \leqq V g_{n}+(1 / n) V h$ on $\operatorname{supp}\left(f^{\prime}\right)$. Proposition 12 gives that $V f^{\prime} \leqq V g_{n}+(1 / n) V h \leqq V g+(1 / n) V h$ on $X$. Letting $f^{\prime} \uparrow f$ and $n \uparrow \infty$, we have $V f \leqq V g$ on $X$.

Similarly as in Definition 11, we define the domination principle for a diffusion kernel.

Definition 14. A diffusion kernel $V$ on $X$ is said to satisfy the domination principle if, for any $\mu, \nu \in M_{K}^{+}(X), V \mu \leqq V \nu$ in a certain neighborhood of $\operatorname{supp}(\mu)$ implies that the same inequality holds on $X^{11}$.

Proposition 15. Let $\left(T_{t}\right)_{t \geqq 0}$ be a transient diffusion semi-group on $X$ and $V$ be the Hunt diffusion kernel for $\left(T_{t}\right)_{t \geqq 0}$. Then $V$ satisfies the domination principle.

Proof. Assume that, for $\mu, \nu \in M_{K}^{+}(X), V \mu \leqq V \nu$ in a certain open neighborhood $\omega$ of $\operatorname{supp}(\mu)$. Choose a relatively compact open set $\omega_{1}$ in $X$ such that $\operatorname{supp}(\mu) \subset \omega_{1} \subset \bar{\omega}_{1} \subset \omega$. Let $\left(V_{p}\right)_{p \geqq 0}$ be the resolvent for $\left(T_{t}\right)_{t \geqq 0}$, and put $\mu_{p}=p V_{p} \mu$ in $\omega_{1}$ and $\mu_{p}=0$ on $C \omega_{1}(p>0)$. Since $\lim _{p \rightarrow \infty} p V_{p} \mu=\mu, \lim _{p \rightarrow \infty} \mu_{p}=\mu$ in $M_{K}(X)$. Hence $\lim _{p \rightarrow \infty} V \mu_{p}=V \mu$ in $M(X)$. By $p(V+(1 / p) I) \cdot V_{p}=V$, we have $(V+(1 / p) I) \mu_{p} \leqq V \nu$ in $\omega$. Put

$$
\begin{gathered}
\lambda=\frac{1}{2}\left(V \nu+\left(V+\frac{1}{p} I\right) \mu_{p}-\left|V \nu-\left(V+\frac{1}{p} I\right) \mu_{p}\right|\right) \\
\left(=\inf \left(V \nu,\left(V+\frac{1}{p} I\right) \mu_{p}\right)\right) .
\end{gathered}
$$

Since $(V+(1 / p) I) \mu_{p} \geqq p V_{p} \lambda$ and $V \nu \geqq p V_{p} \lambda$, we have

$$
\lambda \geqq p V_{p} \lambda \text { and } \lambda=p\left(V+\frac{1}{p} I\right)\left(\lambda-p V_{p} \lambda\right) \text {. }
$$

Since

$$
\begin{aligned}
(I- & \left.p V_{p}\right)\left(\lambda-\left(V+\frac{1}{p} I\right) \mu_{p}\right) \\
& =p V_{p}\left(\left(V+\frac{1}{p} I\right) \mu_{p}-\lambda\right) \leqq 0 \text { in } \omega,
\end{aligned}
$$

1) We denote also by $\operatorname{supp}(\mu)$ the support of $\mu$. 
we have $\lambda \geqq(V+(1 / p) I) \mu_{p}$ on $X$, i.e., $\lambda=(V+(1 / p) I) \mu_{p}$, so that

$$
\left(V+\frac{1}{p} I\right) \mu_{p} \leqq V \nu \text { on } X \text {. }
$$

Letting $p \rightarrow \infty$, we have $V \mu \leqq V \nu$ on $X$. This completes the proof.

Propositions 12, 15 and the Choquet-Deny theorem ${ }^{2)}$ implies the following

Proposition 16. Let $\left(T_{t}\right)_{t \geqq 0}$ be a transient diffusion semi-group on $X$ and $V$ be the Hunt diffusion kernel for $\left(T_{t}\right)_{t \geq 0}$. For any $\mu \in \mathscr{D}^{+}(V)$ and any relatively compact open set $\omega$ in $X$, there exists one and only one $\mu_{\omega}^{\prime} \in M_{K}^{+}(X)$ such that:

$$
\begin{gathered}
\operatorname{supp}\left(\mu_{\omega}^{\prime}\right) \subset \bar{\omega} . \\
V \mu_{\omega}^{\prime} \leqq V \mu \text { on } X . \\
V \mu_{\omega}^{\prime}=V \mu \text { in } \omega .
\end{gathered}
$$

$$
\text { If } \nu \in M_{K}^{+}(X) \text { satisfies } V \nu \geqq V \mu \text { in } \omega \text {, then } V \nu \geqq V \mu_{\omega}^{\prime} \text { on } X \text {. }
$$

Proof. First we assume that $\mu \in M_{K}^{+}(X)$. Choose an exhaustion $\left(\omega_{n}\right)_{n=1}^{\infty}$ of $\omega^{3)}$. The Choquet-Deny theorem ${ }^{2)}$ (see [4]) and Proposition 12 give that there exists $\mu_{n}^{\prime} \in M_{K}^{+}(X)$ such that $\operatorname{supp}\left(\mu_{n}^{\prime}\right) \subset \bar{\omega}_{n}, V \mu_{n}^{\prime} \leqq V \mu$ on $X$ and $V \mu_{n}^{\prime}=V \mu$ in $\omega_{n}$. By Proposition 15, $\left(V \mu_{n}^{\prime}\right)_{n=1}^{\infty}$ is increasing. Since, for any compact $K$ in $X$, there exists $h \in C_{K}^{+}(X)$ such that $V^{*} h(x)>0$ on $K$, $\left(\mu_{n}^{\prime}\right)_{n=1}^{\infty}$ is vaguely bounded, and hence we may assume that it converges vaguely to $\mu_{\omega}^{\prime} \in M_{K}^{+}(X)$ as $n \rightarrow \infty$. We shall show that $\mu_{\omega}^{\prime}$ is a required measure. Evidently $\mu_{\omega}^{\prime}$ satisfies (2.23), (2.24) and (2.25), because $V \mu_{\omega}^{\prime}=$ $\lim _{n \rightarrow \infty} V \mu_{n}^{\prime}$. Let $\nu \in M_{K}^{+}(X)$ satisfy $V \nu \geqq V \mu$ in $\omega$. Then, for any $n \geqq 1$, Proposition 15 gives that $V \mu_{n}^{\prime} \leqq V \nu$ on $X$, so that $V \mu_{\omega}^{\prime} \leqq V \nu$ on $X$, i.e., $\mu_{\omega}^{\prime}$ is a required measure.

In general, we assume that $\mu \in \mathscr{D}^{+}(V)$. We can write $\mu=\sum_{n=1}^{\infty} \mu_{n}$, where $\mu_{n} \in M_{K}^{+}(X)$. Let $\mu_{n, \omega}^{\prime}$ the non-negative Radon measure obtained above for $\mu_{n}$. Then $\sum_{n=1}^{\infty} \mu_{n, \omega}^{\prime}$ converges vaguely. Putting $\mu_{\omega}^{\prime}=\sum_{n=1}^{\infty} \mu_{n, \omega}^{\prime}$, we see easily that $\mu_{\omega}^{\prime}$ is a required measure.

2) This shows that $V^{*}$ satisfies the domination principle if and only if, for any $\mu \in M_{K}^{+}(X)$ and any relatively compact open set $\omega$ in $X$, there exists $\mu^{\prime} \in M_{K}^{+}(X)$ satisfying (2.23), (2.24) and (2.25) in Proposition 16.

3) For an open set $\omega$ in $X,\left(\omega_{n}\right)_{n=1}^{\infty}$ is called an exhaustion of $\omega$ if, for each $n \geqq 1$, $\omega_{n}$ is a relatively compact open set in $\omega, \bar{\omega}_{n} \subset \omega_{n+1}(n=1,2, \cdots)$ and $\cup_{n=1}^{\infty} \omega_{n}=\omega$. 
Finally we show the unicity of $\mu_{\omega}^{\prime}$. Let $\mu_{\omega}^{\prime \prime}$ be another non-negative Radon measure satisfying the required four conditions. Then $V \mu_{\omega}^{\prime}=V \mu_{\omega}^{\prime \prime}$. By virtue of the resolvent equation, we have, for any $p>0, V_{p} \mu_{\omega}^{\prime}=V_{p} \mu_{\omega}^{\prime \prime}$. By remarking that mappings $t \rightarrow T_{t} \mu_{\omega}^{\prime}$ and $t \rightarrow T_{t} \mu_{\omega}^{\prime \prime}$ are vaguely continuous and that the Laplace transformation is injective, we obtain that, for any $t \geqq 0, T_{t} \mu_{\omega}^{\prime}=T_{t} \mu_{\omega}^{\prime \prime}$, i.e., $\mu_{\omega}^{\prime}=\mu_{\omega}^{\prime \prime}$. Thus the unicity of $\mu_{\omega}^{\prime}$ is shown. This completes the proof.

The above non-negative Radon measure $\mu_{\omega}^{\prime}$ is called the $V$-balayaged measure of $\mu$ on $\omega$. In general, the above assertion does not hold if $\omega$ is not relatively compact. Proposition 16 gives the following

CoRollary 17. Let $\left(T_{t}\right)_{t \geqq 0}$ and $V$ be the same as above. The mapping $V: \mathscr{D}(V) \ni \mu \rightarrow V \mu \in M(X)$ is injective.

Proof. Assume that, for $\mu_{j} \in \mathscr{D}^{+}(V)(j=1,2), V \mu_{1}=V \mu_{2}$. Let $\left(\omega_{n}\right)_{n=1}^{\infty}$ be an exhaustion of $X$. Put $\mu_{j, n}=\mu_{j}$ in $\omega_{n}$ and $\mu_{j, n}=0$ on $C \omega_{n}(j=1,2$; $n=1,2, \cdots)$. We denote by $\mu_{j, n}^{\prime \prime}$ the $V$-balayaged measure of $\mu_{j}-\mu_{j, n}$ on $\omega_{n}$. Then $\mu_{j, n}+\mu_{j, n}^{\prime \prime}$ is the $V$-balayaged measure of $\mu_{j}$ on $\omega_{n}(j=1,2$; $n=1,2, \cdots)$. Evidently we have $V\left(\mu_{1, n}+\mu_{1, n}^{\prime \prime}\right)=V\left(\mu_{2, n}+\mu_{2, n}^{\prime \prime}\right)$ for all $n \geqq 1$. In the same manner as above, we have

$$
\mu_{1, n}+\mu_{1, n}^{\prime \prime}=\mu_{2, n}+\mu_{2, n}^{\prime \prime}(n=1,2, \cdots) .
$$

Since $V \mu_{j, n}^{\prime \prime} \leqq V\left(\mu_{j}-\mu_{j, n}\right)$ and $\lim _{n \rightarrow \infty} V\left(\mu_{j}-\mu_{j, n}\right)=0$, we have $\lim _{n \rightarrow \infty} V \mu_{j, n}^{\prime \prime}$ $=0$ (vaguely), and hence $\lim _{n \rightarrow \infty} \mu_{j, n}^{\prime \prime}=0$ (vaguely) for $j=1,2$. Letting $n \rightarrow \infty$ in (2.27), we obtain that $\mu_{1}=\mu_{2}$. This completes the proof.

By generalizing the notion of associated families (see [7]), we define the following

Definition 18. Let $\left(T_{t}\right)_{t \geq 0}$ be a transient continuous semi-group on $X$ and $V$ be the Hunt continuous kernel for $\left(T_{t}\right)_{t \geq 0}$. We say that $\left(T_{t}\right)_{t \geq 0}$ satisfies the condition $(D)$ if, for any $f \in C_{K}^{+}(X)$, there exists an associated family of $f$ with respect to $\left(T_{t}\right)_{t \geq 0}$.

Here, an associated family $\left(f_{n}\right)_{n=1}^{\infty}$ of $f$ with respect to $\left(T_{t}\right)_{t \geqq 0}$ is, by definition, a sequence in $\mathscr{D}^{+}\left(\left(T_{t}\right)_{t \geq 0}\right) \cap \mathscr{D}^{+}(V)$ satisfying the following two conditions:

$$
V f-V f_{n} \in C_{K}^{+}(X)(n=1,2, \cdots) .
$$

$\left(V f_{n}\right)_{n=1}^{\infty}$ converges decreasingly to 0 as $n \uparrow \infty$. 
By the Dini theorem, the convergence in (2.29) is that in the sense of $C(X)$.

Definition 19. Let $\left(T_{t}\right)_{t \geq 0}$ be a transient diffusion semi-group on $X$. We say that $\left(T_{t}\right)_{t \geqq 0}$ satisfies the condition $\left(D^{*}\right)$ if $\left(T_{t}^{*}\right)_{t \geqq 0}$ satisfies the condition $(D)$.

We denote by $\Re(x)$ the totality of compact neighborhoods of $x \in X$.

Proposition 20. Let $\left(T_{t}\right)_{t \geqq 0}$ be a transient diffusion semi-group on $X$ and $V$ be the Hunt diffusion kernel for $\left(T_{t}\right)_{t \geqq 0}$. Assume that $\left(T_{t}\right)_{t \geqq 0}$ satisfies the condition $\left(D^{*}\right)$. Then, for any $\mu \in \mathscr{D}^{+}(V)$ and any $x \in X$,

$$
\bigcap_{N \in \Re(x)} P_{C N}(V ; V \mu)=\{0\},
$$

where $P_{C N}(V ; V \mu)$ denotes the vague closure of the set

$$
\left\{V_{\nu ; \nu} \in M_{K}^{+}(X), \operatorname{supp}(\nu) \subset C N, V_{\nu} \leqq V \mu \text { in } C N\right\} .
$$

Proof. Let $N \in \Re(x)$ and choose an exhaustion $\left(\omega_{n}\right)_{n=1}^{\infty}$ of $C N$. Let $\mu_{n}^{\prime}$ be the $V$-balayaged measure of $\mu$ on $\omega_{n}$. Since $\left(V \mu_{n}^{\prime}\right)_{n=1}^{\infty}$ is increasing and $V \mu_{n}^{\prime} \leqq V \mu$ on $X(n=1,2, \cdots)$,

$$
\eta_{C N}=\lim _{n \rightarrow \infty} V \mu_{n}^{\prime} \quad \text { (vaguely) }
$$

exists. Proposition 15 gives that $\eta_{C N}$ does not depend on the choice of $\left(\omega_{n}\right)_{n=1}^{\infty}$ and that, for any $\eta \in P_{C N}(V ; V \mu), \eta \leqq \eta_{C N}$ on $X$. Choose a sequence $\left(N_{n}\right)_{n=1}^{\infty} \subset \mathfrak{R}(x)$ such that $N_{n} \subset \stackrel{\circ}{N}_{n+1}$ and $\bigcup_{n=1}^{\infty} N_{n}=X$, where $\stackrel{\circ}{N}_{n+1}$ denotes the interior of $N_{n+1}$. Proposition 15 gives that $\left(\eta_{C N_{n}}\right)_{n=1}^{\infty}$ is also decreasing. Put

$$
\eta_{0}=\lim _{n \rightarrow \infty} \eta_{C N_{n}}
$$

Then $\eta_{0} \in \bigcap_{N \in \Re(x)} P_{C N}(V ; V \mu)$ and, for any $\eta^{\prime} \in \bigcap_{N \in \Re(x)} P_{C N}(V ; V \mu), \eta^{\prime} \leqq \eta_{0}$ on $X$. Let $\left(\omega_{n, k}\right)_{k=1}^{\infty}$ be an exhaustion of $C N_{n}$ and $\mu_{n, k}^{\prime}$ be the $V$-balayaged measure of $\mu$ on $\omega_{n, k}(n=1,2, \cdots ; k=1,2, \cdots)$. For any $f \in C_{K}^{+}(X)$ and any associated family $\left(f_{m}\right)_{m=1}^{\infty}$ of $f$ with respect to $\left(T_{t}^{*}\right)_{t \geqq 0}$, we have, for any $m \geqq 1$,

$$
\begin{aligned}
0 & \leqq \int f \eta_{0}=\lim _{n \rightarrow \infty} \int\left(f-f_{m}\right) d \eta_{C N_{n}}+\lim _{n \rightarrow \infty} \int f_{m} d \eta_{C N_{n}} \\
& \leqq \lim _{n \rightarrow \infty} \lim _{k \rightarrow \infty} \int\left(f-f_{m}\right) d V \mu_{n, k}^{\prime}+\int f_{m} d V \mu
\end{aligned}
$$




$$
=\lim _{n \rightarrow \infty} \lim _{k \rightarrow \infty} \int\left(V^{*} f-V^{*} f_{m}\right) d \mu_{n, k}^{\prime}+\int V^{*} f_{m} d \mu \leqq \int V^{*} f_{m} d \mu .
$$

Since $V^{*} f_{m} \leqq V^{*} f$, (2.29) gives that $\lim _{m \rightarrow \infty} \int V^{*} f_{m} d \mu=0$, which implies that $\int f d \eta_{0}=0$. Thus $\eta_{0}=0$, and hence our required equality (2.30) holds. This completes the proof.

Let $\left(T_{t}\right)_{t \geq 0}$ be a transient diffusion semi-group on $X$ and $V$ be the Hunt diffusion kernel for $\left(T_{t}\right)_{t \geq 0}$. For $\lambda \in M(X)$ and an open set $\omega$ in $X$, we put

$$
P_{\omega}(V ; \lambda)=\overline{\left\{V \nu ; \nu \in M_{K}^{+}(X), \operatorname{supp}(\nu) \subset \omega, V \nu \leqq|\lambda| \text { in } \omega\right\}},
$$

where the closure is in the sense of vague topology.

Definition 21. Let $\left(T_{t}\right)_{t \geq 0}$ be a transient diffusion semi-group on $X$ and $V$ be the Hunt diffusion kernel for $\left(T_{t}\right)_{t \geq t}$. We say that $\lambda \in M(X)$ vanishes $V$-n.e. on the boundary of $X$ if, for any $x \in X$,

$$
\bigcap_{N \in \Re(x)} P_{C N}(V ; \lambda)=\{0\}
$$

and if there exists $\mu \in \mathscr{D}^{+}(V)$ such that $|\lambda| \leqq V \mu$.

Evidently, for any $x \in X,(2.36)$ holds if and only if there exists an $x \in X$ satisfying (2.36).

Definition 22. A transient diffusion semi-group $\left(T_{t}\right)_{t \geq 0}$ on $X$ is said to be weakly regular if, for each $\mu \in M_{K}^{+}(X), V \mu$ vanishes $V$-n.e. on the boundary of $X$, where $V$ is the Hunt diffusion kernel for $\left(T_{t}\right)_{t \geq 0}$.

Proposition 23. Let $\left(T_{t}\right)_{t \geq 0}$ be a transient diffusion semi-group on $X$ and $V$ be the Hunt diffusion kernel for $\left(T_{t}\right)_{t \geq 0}$. Then the following two statements are equivalent:

(1) $\left(T_{t}\right)_{t \geq 0}$ is weakly regular.

(2) For any $\mu \in \mathscr{D}^{+}(V)$ and any open set $\omega$ in $X$, there exists one and only one V-balayaged measure $\mu_{\omega}^{\prime}$ of $\mu$ on $\omega^{4)}$. Furthermore we have, for any $x \in X$,

$$
\lim _{\substack{N \in \mathbb{X} \\ N \in \Re(x)}} V \mu_{C N}^{\prime}=0 \text { (vaguely). }
$$

4) This means also a positive Radon measure satisfying the analogous conditions to $(2.23)-(2.26)$. 
Proof. It suffices to show that $(1) \Rightarrow(2)$, because the domination principle for $V$ implies that, for any $N \in \mathfrak{N}(x)$ and any $\eta \in P_{C N}(V ; V \mu), \eta \leqq V \mu_{C N}^{\prime}$ on $X$, and (2.37) gives that $\bigcap_{N \in \mathfrak{M}(x)} P_{C N}(V ; V \mu)=\{0\}$.

Let $x \in X$ and choose a suquence $\left(N_{n}\right)_{n=1}^{\infty} \subset \mathfrak{N}(x)$ such that $N_{n} \subset \stackrel{N}{n+1}_{n}$ and $\bigcup_{n=1}^{\infty} N_{n}=X$. Then $\left(\eta_{C N_{n}}\right)_{n=1}^{\infty}$ is decreasing. Since $\eta_{C N_{n}} \in P_{C N_{n}}(V ; V \mu)$, the weak regularity of $V$ gives that $\lim _{n \rightarrow \infty} \eta_{C N_{n}}=0$ (vaguely). Similarly as in Proposition 16, it suffices to assume that $\mu \in M_{K}^{+}(X)$. Let $\left(\omega_{n}\right)_{n=1}^{\infty}$ be an exhaustion of $\omega$ and $\mu_{n}^{\prime}$ be the $V$-balayaged measure of $\mu$ on $\omega_{n}$. Then $\left(V \mu_{n}^{\prime}\right)_{n=1}^{\infty}$ is increasing and $V \mu_{n}^{\prime} \leqq V \mu$ on $X(n=1,2, \cdots)$. Put

$$
\eta_{\omega}=\lim _{n \rightarrow \infty} V \mu_{n}^{\prime} \text {. }
$$

Then $\eta_{\omega} \in P_{\omega}(V ; V \mu)$ and $\eta_{\omega}$ does not depend on the choice of $\left(\omega_{n}\right)_{n=1}^{\infty}$. Since $\left(\mu_{n}^{\prime}\right)_{n=1}^{\infty}$ is vaguely bounded, we may assume that it converges vaguely to $\mu_{\omega}^{\prime} \in M^{+}(X)$ as $n \rightarrow \infty$. Evidently $\eta_{\omega} \geqq V \mu_{\omega}^{\prime}$ on $X$. We shall show the inverse inequality. Let $\varphi_{k} \in C_{K}^{+}(X)$ such that $0 \leqq \varphi_{k} \leqq 1, \varphi_{k}=1$ on $N_{k}$ and $\operatorname{supp}\left(\varphi_{k}\right) \subset \stackrel{\circ}{N}_{k+1}(k=1,2, \cdots)$. Then, for any $n \geqq 1, V\left(\left(1-\varphi_{k+1}\right) \mu_{n}^{\prime}\right)$ $\in P_{C N_{k}}(V ; V \mu)(k=1,2, \cdots)$, and hence $V\left(\left(1-\varphi_{k+1}\right) \mu_{n}^{\prime}\right) \leqq \eta_{C N_{k}}$ on $X$. Therefore, for any $f \in C_{K}^{+}(X)$,

$$
\begin{array}{r}
\int f d V \mu_{\omega}^{\prime} \geqq \int f d V\left(\varphi_{k+1} \mu_{\omega}^{\prime}\right)=\lim _{n \rightarrow \infty} \int f d V\left(\varphi_{k+1} \mu_{n}^{\prime}\right) \geqq \int f d \eta_{\omega}-\int f d \eta_{C N_{k}} \\
(k=1,2, \cdots) .
\end{array}
$$

Letting $k \rightarrow \infty$, we obtain that $V \mu_{\omega}^{\prime} \geqq \eta_{\omega}$ on $X$. Thus $\eta_{\omega}=V \mu_{\omega}^{\prime}$. Similarly as in Proposition 16, $\mu_{\omega}^{\prime}$ is a required measure. Its unicity follows directly from Corollary 17.

Let $\left(T_{t}\right)_{t \geqq 0}$ be a transient diffusion semi-group on $X$ and $V$ be a Hunt diffusion kernel for $\left(T_{t}\right)_{t \geq 0}$. Put

$$
R\left(V^{*}\right)=\left\{V^{*} f ; f \in \mathscr{D}\left(\left(T_{t}^{*}\right)_{t \geqq 0}\right) \cap \mathscr{D}\left(V^{*}\right)\right\},
$$

$R^{+}\left(V^{*}\right)=R\left(V^{*}\right) \cap C^{+}(X), R_{K}\left(V^{*}\right)=R\left(V^{*}\right) \cap C_{K}(X)$ and $R_{K}^{+}\left(V^{*}\right)=R\left(V^{*}\right)$ $\cap C_{K}^{+}(X)$. Then $R_{K}\left(V^{*}\right)$ is a linear subspace of $C_{K}(X)$ and $R_{K}^{+}\left(V^{*}\right)$ is a convex cone. Put

$$
\mathscr{D}^{0}=\left\{\mu \in M(X) ; \int|f| d|\mu|<\infty \text { for any } V^{*} f \in R_{K}\left(V^{*}\right)\right\}
$$

and, for each $\mu \in \mathscr{D}^{0}$, define the linear functional $A \mu$ on $R_{K}\left(V^{*}\right)$ by

$$
A \mu\left(V^{*} f\right)=-\int f d \mu \text { for any } V^{*} f \in R_{K}\left(V^{*}\right) .
$$


Precisely we write $\mathscr{D}^{0}(A)=\mathscr{D}^{0}$. Then we have easily the following

Remark 24. Let $\left(T_{t}\right)_{t \geqq 0}$ and $V$ be the same as above. Assume that $R_{K}^{+}\left(V^{*}\right)$ is total in $C_{K}(X)^{5)}$. Then, for $\mu \in \mathscr{D}^{0}$, a continuous extension of $A \mu$ to $C_{K}(X)$ is uniquely determined if it exists. Furthermore if, for $\mu \in \mathscr{D}^{0}$, $-A \mu$ is non-negative, i.e., $-A \mu(g) \geqq 0$ if $g \in R_{K}^{+}\left(V^{*}\right)$, then a positive linear extension of $-A \mu$ to $C_{K}(X)$ exists.

Definition 25. Let $\left(T_{t}\right)_{t \geqq 0}$ be a transient diffusion semi-group on $X$ and $V$ be the Hunt diffusion kernel for $\left(T_{t}\right)_{t \geq 0}$. If $R_{K}^{+}\left(V^{*}\right)$ is total in $C_{K}(X)$, then $\left(T_{t}\right)_{t \geqq 0}$ is said to satisfy the condition $\left(C^{*}\right)$.

For a transient diffusion semi-group on $X$ satisfying the condition $\left(C^{*}\right)$, we denote by $\mathscr{D}(A)$ the set of all $\mu \in \mathscr{D}^{0}(A)$ such that a continuous linear extension to $C_{K}(X)$ exists. For $\mu \in \mathscr{D}(A)$, we can write again $A \mu$ its continuous linear extension to $C_{K}(X)$ without confusion (see Remark 24). Evidently $\mathscr{D}(A)$ is a linear subspace of $M(X)$ and the linear operator $A: \mathscr{D}(A) \ni \mu \rightarrow A \mu \in M(X)$ is defined.

Definition 26. The above linear operator $A$ is called the infinitesimal generator of $\left(T_{t}\right)_{t \geq 0}$.

Definition 27. Let $\left(T_{t}\right)_{t \geqq 0}$ be a transient diffusion semi-group on $X$. If $\left(T_{t}\right)_{t \geqq 0}$ satisfies the conditions $\left(D^{*}\right)$ and $\left(C^{*}\right)$, it is said to be regular.

If a transient diffusion semi-group $\left(T_{t}\right)_{t \geqq 0}$ is of convolution type, it is always regular (see, for example, [7] and [8]).

Remark 28. Let $\left(T_{t}\right)_{t \geqq 0}$ be a transient diffusion semi-group on $X$ and $\left(V_{p}\right)_{p \geqq 0}$ be the resolvent for $\left(T_{t}\right)_{t \geqq 0}$. Let $p>0$ and put

$$
T_{p, t}=\exp (-p t)\left(I+\sum_{n=1}^{\infty} \frac{(p t)^{n}}{n !}\left(p V_{p}\right)^{n}\right)(t>0) \text { and } T_{p, 0}=I \text {. }
$$

Then $\left(T_{p, t}\right)_{t \geq 0}$ is a transient diffusion semi-group on $X$ and $V+(1 / p) I=$ $\int_{0}^{\infty} T_{p, t} d t$, where $V_{0}=V$. Furthermore, if $\left(T_{t}\right)_{t \geqq 0}$ is regular (resp. weakly regular), then so is $\left(T_{p, t}\right)_{t \geqq 0}$ for any $p>0$.

In fact, (2.13) gives directly the first part. Assume that $\left(T_{t}\right)_{t \geqq 0}$ is regular. Since $p\left(V^{*}+(1 / p) I\right) \cdot\left(I-p V_{p}^{*}\right)=I, C_{K}(X)=R_{K}\left(V^{*}+(1 / p) I\right)$, and hence $\left(T_{p, t}\right)_{t \geqq 0}$ satisfies the condition $\left(C^{*}\right)$. Let $f \in C_{K}^{+}(X)$ and $\left(f_{n}\right)_{n=1}^{\infty}$ be an

5) This means that $R_{K}^{+}\left(V^{*}\right) \subset C_{K}(X)$ and, for any $x \in X$ and any neighborhood $U$ of $x$, there exists an $f \neq 0 \in R_{K}^{+}\left(V^{*}\right)$ such that $\operatorname{supp}(f) \subset U$. 
associated family of $f$ with respect to $\left(T_{t}^{*}\right)_{t \geq 0 .}$. Then $p V_{p}^{*} f_{n} \in \mathscr{D}\left(\left(T_{p, t}^{*}\right)_{t \geq 0}\right) \cap$ $\mathscr{D}\left(V^{*}+(1 / p) I\right)$ and $\left(V^{*}+(1 / p) I\right)\left(p V_{p}^{*} f_{n}\right)=V^{*} f_{n}$. Thus we see that $\left(p V_{p}^{*} f_{n}\right)_{n=1}^{\infty}$ is an associated family of $f$ with respect to $\left(T_{p, t}^{*}\right)_{t \geq 0}$. Hence $\left(T_{p, t} t_{t \geq 0}\right.$ is regular for any $p>0$. Next we assume that $V$ is weakly regular. Let $p>0$ be fixed and $\mu \in M_{\bar{K}}^{+}(X)$. For any $x \in X$ and any $N \in \mathfrak{R}(x)$ with $\stackrel{\circ}{\supset} \operatorname{supp}(\mu)$, we have, in the same manner as in Proposition 15,

$$
\left(V+\frac{1}{p} I\right) \nu \leqq V \mu_{C N}^{\prime} \text { on } X
$$

whenever $(V+(1 / p) I) \nu \in P_{C N}(V+(1 / p) I ;(V+(1 / p) I) \mu)$, where $\mu_{C N}^{\prime}$ is the $V$-balayaged measure of $\mu$ on $C N$. By Proposition 23 and (2.44), $V+(1 / p) I$ is weakly regular.

Remark 29. Let $\left(T_{t}\right)_{t \geq 0}$ be a transient diffusion semi-group on $X$ satisfying the condition $\left(C^{*}\right), V$ be the Hunt diffusion kernel for $\left(T_{t}\right)_{t \geqq 0}$ and $A$ be the infinitesimal generator of $\left(T_{t}\right)_{t \geq 0}$. Then, for any $\mu \in \mathscr{D}(V), V \mu \epsilon$ $\mathscr{D}(A)$ and $A(V \mu)=-\mu$.

In fact, we may assume that $\mu$ is non-negative. For any $V^{*} f \in R_{K}^{+}\left(V^{*}\right)$,

$$
\lim _{t \rightarrow 0} \frac{1}{t}\left(I-T_{t}^{*}\right)\left(V^{*} f\right)=\lim _{t \rightarrow 0} \frac{1}{t} \int_{0}^{t} T_{s}^{*} f d s=f \text { (pointwise). }
$$

Since $\operatorname{supp}\left(f^{+}\right) \subset \operatorname{supp}\left(V^{*} f\right)$,

$$
\int|f| d V \mu \leqq 2 \int f^{+} d V \mu<\infty,
$$

which gives that $V \mu \in \mathscr{D}^{0}(A)$, because, for any $V^{*} f \in R_{K}\left(V^{*}\right)$, there exists $V^{*} g \in R_{K}^{+}\left(V^{*}\right)$ such that $V^{*} g \geqq\left|V^{*} f\right|$. Since, for any $V^{*} f \in R_{K}\left(V^{*}\right)$, $\int V^{*} f d \mu=\int f d V \mu$, our assertion holds.

\section{§ 3. The Riesz decomposition theorem}

We begin by the following two lemmas:

Lemma 30. Let $\left(T_{t}\right)_{t \geqq 0}$ be a transient diffusion semi-group on $X$ and $V$ be the Hunt diffusion kernel for $\left(T_{t}\right)_{t \geq 0}$. For a given positive Radon measure $\mu$ in $X$, there exists $h \in \mathscr{D}^{+}\left(\left(T_{t}^{*}\right)_{t \geq 0}\right) \cap \mathscr{D}^{+}\left(V^{*}\right)$ such that $V^{*} h(x)>0$ on $X$ and $\int h d \mu<\infty$.

Proof. Let $\left(\omega_{n}\right)_{n=1}^{\infty}$ be an exhaustion of $X$. Then, for any $n$, there 
exists $h_{n} \in C_{K}^{+}(X)$ such that $V^{*} h_{n}>0$ in $\omega_{n}$. We choose also $g_{n} \in C_{K}^{+}(X)$ satisfying $V^{*} g_{n} \geqq h_{n}$ on $X$. Since, for any $t>0$,

$$
0 \leqq T_{t}^{*} h_{n} \leqq T_{t}^{*}\left(V^{*} g_{n}\right)=\int_{t}^{\infty} T_{s}^{*} g_{n} d s \leqq V^{*} g_{n} \text { on } X,
$$

there exists a constant $c_{n}>0$ such that

$$
\begin{aligned}
c_{n} V^{*} h_{n} \leqq \frac{1}{2^{n}}, c_{n} T_{t}^{*} h_{n} \leqq \frac{1}{2^{n}} \text { on } \bar{\omega}_{n}(0 \leqq t<\infty) \\
\quad \text { and } c_{n} \int h_{n} d \mu<\frac{1}{2^{n}} .
\end{aligned}
$$

Then $h=\sum_{n=1}^{\infty} c_{n} h_{n}$ is a required function.

Lemma 31. Let $\left(T_{t}\right)_{t \geq 0}$ be a transient diffusion semi-group on $X$ satisfying the condition $\left(D^{*}\right)$ and $V$ be the Hunt diffusion kernel for $\left(T_{t}\right)_{t \geq 0}$. For any $f \in \mathscr{D}^{+}\left(\left(T_{t}^{*}\right)_{t \geq 0}\right) \cap \mathscr{D}^{+}\left(V^{*}\right)$, there exists also an associated family of $f$ with respect to $\left(T_{t}^{*}\right)_{t \geqq 0}$.

Proof. Choose a sequence $\left(f_{n}\right)_{n=1}^{\infty} \subset C_{K}^{+}(X)$ such that $f=\sum_{n=1}^{\infty} f_{n}$ and an exhaustion $\left(\omega_{n}\right)_{n=1}^{\infty}$ of $X$. Let $\left(f_{n, m}\right)_{m=1}^{\infty}$ be an associated family of $f_{n}$ with respect to $\left(T_{t}^{*}\right)_{t \geq 0}$. We may assume that, for any $m \geqq 1$ and any $k$ with $1 \leqq k \leqq m, V^{*} f_{k, m} \leqq 1 / m^{2}$ on $\bar{\omega}_{m}$. Put

$$
g_{n}=\sum_{k=1}^{n} f_{k, n}+\sum_{k=n+1}^{\infty} f_{k}(n=1,2, \cdots),
$$

then $g_{n} \in \mathscr{D}\left(\left(T_{t}^{*}\right)_{t \geqq 0}\right) \cap \mathscr{D}\left(V^{*}\right)$. We see easily that $\left(g_{n}\right)_{n=1}^{\infty}$ is a required associated family of $f$ with respect to $\left(T_{t}^{*}\right)_{t \geqq 0}$.

Definition 32. Let $\left(T_{t}\right)_{t \geqq 0}$ be a transient diffusion semi-group on $X$ satisfying the condition $\left(C^{*}\right)$ and $A$ be the infinitesimal generator of $\left(T_{t}\right)_{t \geq 0}$. A real Radon measure $\mu$ in $X$ is said to be $A$-superharmonic (resp. $A$-harmonic) if $\mu \in \mathscr{D}(A)$ and $-A \mu \in M^{+}(X)$ (resp. $A \mu=0$ ).

Clearly this is equivalent to $\mu \in \mathscr{D}^{\circ}(A)$ and $\int f d \mu \geqq 0$ (resp. $\left.\int f d \mu=0\right)$ for all $V^{*} f \in R_{K}^{+}\left(V^{*}\right)$, because $R_{K}^{+}\left(V^{*}\right)$ is total in $C_{K}(X)$ and forms a convex cone.

Definition 33. Let $\left(T_{t}\right)_{t \geqq 0}$ be a diffusion semi-group on $X$. A real Radon measure $\mu$ in $X$ is said to be excessive (resp. invariant) with respect to $\left(T_{t}\right)_{t \geqq 0}$ if, for any $t \geqq 0, \mu \in \mathscr{D}\left(T_{t}\right)$ and $\mu \geqq T_{t} \mu$ (resp. $\mu=T_{t} \mu$ ). 
Remark 34. Let $\left(T_{t}\right)_{t \geq 0}$ be a transient diffusion semi-group satisfying the condition $\left(C^{*}\right)$ and $A$ be the infinitesimal generator of $\left(T_{t}\right)_{t \geq 0}$. If $\mu \in M^{+}(X)$ is excessive with respect to $\left(T_{t}\right)_{t \geqq 0}$, then $\mu$ is $A$-superharmonic.

In fact, for $g=V^{*} f \in R_{K}^{+}\left(V^{*}\right)$ and $t>0$, we put $f_{t}^{+}=1 / t\left(g-T_{t}^{*} g\right)^{+}$and $f_{t}^{-}=1 / t\left(g-T_{t}^{*} g\right)^{-}$. Then $\operatorname{supp}\left(f_{t}^{+}\right) \subset \operatorname{supp}(g)$ for all $t>0$, and hence the Lebesgue theorem gives that $\lim _{t \rightarrow 0} \int f_{t}^{+} d \mu=\int f^{+} d \mu$. By the Fatou lemma and $\lim _{t \rightarrow 0} f_{t}^{-}(x)=f^{-}(x)$ for all $x \in X$,

$$
\begin{aligned}
0 & \leqq \varliminf_{t \rightarrow 0} \frac{1}{t} \int g d\left(I-T_{t}\right) \mu=\underline{\varliminf_{t \rightarrow 0}} \frac{1}{t} \int\left(I-T_{t}^{*}\right) g d \mu \\
& =\varliminf_{t \rightarrow 0} \int\left(f_{t}^{+}-f_{t}^{-}\right) d \mu \leqq \int f^{+} d \mu-\int f^{-} d \mu=\int f d \mu,
\end{aligned}
$$

which implies that $\mu$ is $A$-superharmonic.

The main theorem of this paragraph is the following Riesz decomposition theorem.

THEOREM 35. Let $\left(T_{t}\right)_{t \geqq 0}$ be a transient and regular diffusion semi-group on $X, V$ be the Hunt diffusion kernel for $\left(T_{t}\right)_{t \geq 0}$ and $A$ be the infinitesimal generator of $\left(T_{t}\right)_{t \geqq 0}$. Then every non-negative A-superharmonic measure $\mu$ in $X$ can be written uniquely as

$$
\mu=V \nu+\mu_{h}
$$

where $\nu \in \mathscr{D}^{+}(V)$ and $\mu_{n}$ is a non-negative A-harmonic measure in $X$. Furthermore $\nu=-A \mu$.

First we prepare the following two lemmas.

Lemma 36. Let $\left(T_{t}\right)_{t \geq 0}, V$ and $A$ be the same as above, and let $\mu$ be a positive A-superharmonic measure. Then, for any $f \in \mathscr{D}^{+}\left(\left(T_{t}^{*}\right)_{t \geqq 0}\right) \cap \mathscr{D}^{+}\left(V^{*}\right)$ with $\int f d \mu<\infty$ and an associated family $\left(f_{n}\right)_{n=1}^{\infty}$ of $f$ with respect to $\left(T_{t}^{*}\right)_{t \geqq 0}$, $\left(\int f_{n} d \mu\right)_{n=1}^{\infty}$ is decreasing, $\int f_{n} d \mu \leqq \int f d \mu(n=1,2, \cdots)$ and $\lim _{n \rightarrow \infty} \int f_{n} d \mu$ does not depend on the choice of $\left(f_{n}\right)_{n=1}^{\infty}$.

Proof. Since, for any $n \geqq 1, V^{*}\left(f-f_{n}\right) \in R_{K}^{+}\left(V^{*}\right), \int f_{n} d \mu \leqq \int f d \mu$ and $\left(\int f_{n} d \mu\right)_{n=1}^{\infty}$ is decreasing. Let $\left(g_{n}\right)_{n=1}^{\infty}$ be another associated family of $f$ with respect to $\left(T_{t}^{*}\right)_{t \geqq 0}$. We choose $h \in \mathscr{D}^{+}\left(\left(T_{t}^{*}\right)_{t \geqq 0}\right) \cap \mathscr{D}^{+}\left(V^{*}\right)$ satisfying $V^{*} h>0$ 
on $X$ and $\int h d \mu<\infty$ (see Lemma 30) and an associated family $\left(h_{n}\right)_{n=1}^{\infty}$ of $h$ with respect to $\left(T_{t}^{*}\right)_{t \geqq 0}$. For any integer $m \geqq 1$ and any positive number $\delta$, there exists an integer $n_{0} \geqq 1$ such that, for all $n \geqq n_{0}$,

$$
\delta V^{*}\left(h-h_{n}\right)+V^{*} f-V^{*} f_{n} \geqq V^{*} f-V^{*} g_{m} \text { on } X,
$$

which implies that

$$
\int\left(\delta\left(h-h_{n}\right)+g_{m}-f_{n}\right) d \mu \geqq 0 .
$$

Letting $n \rightarrow \infty$ and next $\delta \rightarrow 0, m \rightarrow \infty$, we obtain that

$$
\lim _{m \rightarrow \infty} \int g_{m} d \mu \geqq \lim _{n \rightarrow \infty} \int f_{n} d \mu \text {. }
$$

In the same manner, we see the inverse inequality. Thus $\lim _{n \rightarrow \infty} \int f_{n} d \mu$ does not depend on $\left(f_{n}\right)_{n=1}^{\infty}$, and hence the proof is achieved.

Lemma 37. Let $\left(T_{t}\right)_{t \geqq 0}, V, A$ and $\mu$ be the same as above. Assume that, for any $f \in \mathscr{D}^{+}\left(V^{*}\right)$ with $\int f d \mu<\infty$ and any associated family $\left(f_{n}\right)_{n=1}^{\infty}$ of $f$ with respect to $\left(T_{t}^{*}\right)_{t \geqq 0}, \lim _{n \rightarrow \infty} \int f_{n} d \mu=0$. Then, for any $V^{*} g \in R^{+}\left(V^{*}\right)$, $\int g d \mu \geqq 0$ whenever $\int g^{+} d \mu<\infty$.

Proof. It suffices to show that for any $f \in C_{K}^{+}(X)$ with $f \leqq g^{-}, \int g^{+} d \mu$ $\geqq \int f d \mu$. Let $\left(g_{n}\right)_{n=1}^{\infty}$ and $\left(f_{n}\right)_{n=1}^{\infty}$ be an associated family of $g^{+}$with respect to $\left(T_{t}^{*}\right)_{t \geqq 0}$ and that of $f$ with respect to $\left(T_{t}^{*}\right)_{t \geqq 0}$, respectively. Let $h$ and $\left(h_{n}\right)_{n=1}^{\infty}$ be the same as in the above proof. Similarly as in Lemma 36, for any integer $n \geqq 1$ and any number $\delta>0$, there exists an integer $m_{0} \geqq 1$ such that, for all $m \geqq m_{0}$,

$$
\delta\left(V^{*} h-V^{*} h_{m}\right)+V^{*} g^{+}-V^{*} g_{m} \geqq V^{*} f-V^{*} f_{n} \text { on } X,
$$

and hence

$$
\int \delta\left(h-h_{m}\right) d \mu+\int\left(g^{+}-g_{m}+f_{n}-f\right) d \mu \geqq 0
$$

Letting $m \rightarrow \infty$ and next $\delta \rightarrow 0, n \rightarrow \infty$, we obtain that $\int g^{+} d \mu \geqq \int f d \mu$. 
Thus Lemma 37 is shown.

Proof of Theorem 35. By Lemma 36, there exists one and only one $\mu_{h} \in M^{+}(X)$ such that, for any $f \in C_{K}^{+}(X)$,

$$
\int f d \mu_{h}=\lim _{n \rightarrow \infty} \int f_{n} d \mu
$$

where $\left(f_{n}\right)_{n=1}^{\infty}$ is an associated family of $f$ with respect to $\left(T_{t}^{*}\right)_{t \geqq 0}$. Put $\mu_{p}=\mu-\mu_{h}$. Then we shall show the following two statements:

(a) $\mu_{h}$ is $A$-harmonic.

(b) There exists $\nu \in \mathscr{D}^{+}(V)$ such that $\mu_{p}=V \nu$.

We begin by the proof of (a). Let $V^{*} f \in R_{K}^{+}\left(V^{*}\right)$. Then $|f| \in \mathscr{D}\left(\left(T_{t}^{*}\right)_{t \geqq 0}\right)$ $\cap \mathscr{D}\left(V^{*}\right)$ and $\operatorname{supp}\left(f^{+}\right)$is compact (see the proof of Remark 34). Let $\left(f_{n}\right)_{n=1}^{\infty}$ be an associated family of $f^{-}$with respect to $\left(T_{t}^{*}\right)_{t \geq 0}$. Then it is also an associated family of $f^{+}$with respect to $\left(T_{t}^{*}\right)_{t \geqq 0}$. Hence (a) follows from the equality

$$
\int g d \mu_{n}=\lim _{n \rightarrow \infty} \int g_{n} d \mu
$$

for any $g \in \mathscr{D}^{+}\left(\left(T_{t}^{*}\right)_{t \geqq 0}\right) \cap \mathscr{D}^{+}\left(V^{*}\right)$ with $\int g d \mu<\infty$, where $\left(g_{n}\right)_{n=1}^{\infty}$ is an associated family of $g$ with respect to $\left(T_{t}^{*}\right)_{t \geqq 0}$. We remark that $\int g d \mu_{h} \leqq$ $\int g d \mu$, because, for any $g^{\prime} \in C_{K}^{+}(X)$ with $g^{\prime} \leqq g, \int g^{\prime} d \mu_{h} \leqq \int g^{\prime} d \mu \leqq \int g d \mu$. Let $h$ and $\left(h_{n}\right)_{n=1}^{\infty}$ be the same as in the proof of Lemma 36, and let $\left(f_{n}\right)_{n=1}^{\infty}$ be an increasing sequence $\subset C_{K}^{+}(X)$ with $\lim _{n \rightarrow \infty} f_{n}=g$ in $C(X)$. Then $\left(V^{*} f_{n}\right)_{n=1}^{\infty}$ converges increasingly to $V^{*} g$ as $n \uparrow \infty$, i.e., $\lim _{n \rightarrow \infty} V^{*} f_{n}=V^{*} g$ in $C(X)$. For any integer $n \geqq 1$ and any number $\delta>0$, there exists an integer $m_{0} \geqq 1$ such that, for all $m \geqq m_{0}$,

$$
\delta V^{*} h+V^{*} f_{m}>V^{*} g-V^{*} g_{n} \text { on } X .
$$

Let $\left(f_{n, k}\right)_{k=1}^{\infty}$ be an associated family of $f_{n}$ with respect to $\left(T_{t}^{*}\right)_{t \geq 0}$. By (3.13), for any $m \geqq m_{0}$, there exists $k_{m} \geqq 1$ such that, for all $k \geqq k_{m}$,

$$
\delta V^{*}\left(h-h_{k}\right)+V^{*}\left(f_{m}-f_{m, k}\right) \geqq V^{*} g-V^{*} g_{n} \text { on } X .
$$

This implies that

$$
\delta \int\left(h-h_{k}\right) d \mu+\int\left(f_{m}-f_{m, k}\right) d \mu \geqq \int\left(g-g_{n}\right) d \mu .
$$


Letting $k \rightarrow \infty, m \rightarrow \infty, \delta \rightarrow 0$ and $n \rightarrow \infty$, we obtain that

$$
\int g d \mu_{n} \leqq \lim _{n \rightarrow \infty} \int g_{n} d \mu
$$

On the other hand, for any integer $n \geqq 1, k \geqq 1$ and any positive number $\delta>0$, there exists an integer $m_{0} \geqq 1$ such that, for all $m \geqq m_{0}$,

$$
\delta\left(V^{*} h-V^{*} h_{m}\right)+V^{*}\left(g-g_{m}\right) \geqq V^{*}\left(f_{n}-f_{n, k}\right) \text { on } X .
$$

This gives that the inverse inequality of (3.16) holds, i.e., (3.12) holds. Consequently (a) is shown.

Next we shall show (b). By (a) and (3.12), $\mu_{p}$ is a positive $A$-superharmonic measure and the assumption in Lemma 37 is satisfied. For any $f \in C_{K}^{+}(X)$ and any $t>0, \quad V^{*}\left(I-T_{t}^{*}\right) f=\int_{0}^{t} T_{s}^{*} f d s \in R^{+}\left(V^{*}\right)$ and $\int((I-$ $\left.\left.T_{t}^{*}\right) f\right)^{+} d \mu<\infty$. Hence Lemma 37 gives that

$$
0 \leqq \int\left(I-T_{t}^{*}\right) f d \mu_{p}=\int f d\left(I-T_{t}\right) \mu_{p}
$$

and hence, $\left(I-T_{t}\right) \mu_{p} \in M^{+}(X)$ for any $t>0$. For any $f \in C_{K}^{+}(X)$, we choose $g \in C_{K}^{+}(X)$ such that $f \leqq V^{*} g$ on $X$. Since, for any $t>0$,

$$
\begin{gathered}
\frac{1}{t} \int f d\left(I-T_{t}\right) \mu_{p} \leqq \frac{1}{t} \int V^{*} g d\left(I-T_{t}\right) \mu_{p} \\
=\frac{1}{t} \iint_{0}^{t} T_{s}^{*} g d s d \mu_{p} \leqq \int g d \mu_{p},
\end{gathered}
$$

$\left(1 / t\left(I-T_{t}\right) \mu_{p}\right)_{t>0}$ is vaguely bounded. Let $\nu \in M^{+}(X)$ be its vaguely cluster point as $t \rightarrow 0$ and choose a sequence $\left(t_{n}\right)_{n=1}^{\infty}$ of positive numbers such that $\lim _{n \rightarrow \infty} t_{n}=0$ and $\lim _{n \rightarrow \infty} 1 / t_{n}\left(I-T_{t_{n}}\right) \mu_{p}=\nu$ (vaguely). By remarking (3.19) and $\lim _{t \rightarrow 0} T_{t}=I$, we have $\nu \in \mathscr{D}^{+}(V)$ and $\mu_{p} \geqq V \nu$. On the other hand, let $f \in C_{K}^{+}(X)$ and $\left(f_{n}\right)_{n=1}^{\infty}$ be its associated family with respect to $\left(T_{t}^{*}\right)_{t \geqq 0}$. Then, for any $k \geqq 1$,

$$
\begin{aligned}
\int f d V \nu & =\int V^{*} f d \nu \geqq \int V^{*}\left(f-f_{k}\right) d \nu \\
& =\lim _{n \rightarrow \infty} \int V^{*}\left(f-f_{k}\right) d\left(\frac{1}{t_{n}}\left(I-T_{t_{n}}\right) \mu_{p}\right) \\
& =\lim _{n \rightarrow \infty} \frac{1}{t_{n}} \int_{0}^{t_{n}}\left(\int\left(f-f_{k}\right) d T_{s} \mu_{p}\right) d s \geqq \int f d \mu_{p}-\int f_{k} d \mu_{p},
\end{aligned}
$$

because the vague boundedness of $\left(1 / t\left(I-T_{t}\right) \mu_{p}\right)_{t>0}$ leads to $\lim _{t \rightarrow 0} T_{t} \mu_{p}=\mu_{p}$ 
(vaguely). Letting $k \rightarrow \infty$ in (3.20), we obtain that $\int f d V \nu \geqq \int f d \mu_{p}$, i.e., $V \nu \geqq \mu_{p}$. Thus we have $\mu_{p}=V \nu$. We have also $\lim _{t \rightarrow 0} 1 / t\left(I-T_{t}\right) \mu_{p}=\nu$ (vaguely), by the injectivity of $V$. Consequently we have $\mu=V \nu+\mu_{h}$. Let $\mu=V \nu^{\prime}+\mu_{n}^{\prime}$ be another decomposition satisfying our required conditions. Then Remark 29 implies that $-A \mu=\nu=\nu^{\prime}$, and so $\mu_{h}=\mu_{h}^{\prime}$. Thus we see the unicity of the decomposition of $\mu$ and $\nu=-A \mu$. This completes the proof.

Definition 38. The above $V \nu$ and $\mu_{h}$ are called the potential part of $\mu$ and the harmonic part of $\mu$, respectively. The decomposition of $\mu$ in Theorem 35 is called the Riesz decomposition of $\mu$.

Theorem 35 gives directly the following

Corollary 39. Let $\left(T_{t}\right)_{t \geqq 0}, V$ and $A$ be the same as in Theorem 35. Then we have;

(1) If $\mu \in M^{+}(X)$ is invariant with respect to $\left(T_{t}\right)_{t \geqq 0}$, then $\mu$ is $A$ harmonic.

(2) Let $\mu \in M^{+}(X)$ be A-superharmonic. The harmonic part of $\mu$ is the greatest $A$-harmonic minorant of $\mu$.

Evidently (1) holds. Let $\nu \in M^{+}(X)$ be an $A$-harmonic measure satisfying $\mu \geqq \nu$. Applying Theorem 35 to $\mu-\nu$, we see that $\mu_{h} \geqq \nu$, where $\mu_{h}$ is the harmonic part of $\mu$.

Now we consider $A^{*}$-superharmonic functions and $A^{*}$-harmonic functions.

Definition 40. Let $\left(T_{t}\right)_{t \geqq 0}$ be a transient diffusion semi-group on $X$ satisfying the condition $\left(C^{*}\right), V$ be the Hunt diffusion kernel for $\left(T_{t}\right)_{t \geqq 0}$ and $A$ be the infinitesimal generator of $\left(T_{t}\right)_{t \geqq 0}$. Let $\Omega$ be an open set in $X$. A real-valued Borel function $u$ in $X$ is said to be $A^{*}$-superharmonic (resp. $A^{*}$-harmonic) in $\Omega$ if $\int|u| d|A \mu|<\infty$ and $-\int u d A \mu \geqq 0$ (resp. $\left.\int u d A \mu=0\right)$ for any $\mu \in \mathscr{D}_{K}^{+}(A ; \Omega)$, where

$$
\mathscr{D}_{K}^{+}(A ; \Omega)=\left\{V \mu \in M_{K}^{+}(X) ; \mu \in \mathscr{D}(V) \text { and } \operatorname{supp}(V \mu) \subset \Omega\right\} .
$$

LEMMA 41. Let $\left(T_{t}\right)_{t \geqq 0}$ be a transient and weakly regular diffusion semigroup on $X$ and $V$ be the Hunt diffusion kernel for $\left(T_{t}\right)_{t \geqq 0}$. Let $\mu \in \mathscr{D}^{+}(V)$ and $F$ be a closed set in $X$. For an exhaustion $\left(\omega_{n}\right)_{n=1}^{\infty}$ of $C F$, we denote by $\mu_{n}^{\prime}$ the $V$-balayaged measure of $\mu$ on $C \bar{\omega}_{n}$. Then $\left(\mu_{n}^{\prime}\right)_{n=1}^{\infty}$ converges vaguely 
and its limit does not depend on the choice of $\left(\omega_{n}\right)_{n=1}^{\infty}$.

Proof. Evidently $\left(V \mu_{n}^{\prime}\right)_{n=1}^{\infty}$ is decreasing and $V \mu_{n}^{\prime} \leqq V \mu$. This implies also that $\left(\mu_{n}^{\prime}\right)_{n=1}^{\infty}$ is vaguely bounded. Let $\mu_{F}^{\prime}$ be its vaguely cluster point as $n \rightarrow \infty$. Similarly as in Proposition 23, we have

$$
V \mu_{F}^{\prime}=\lim _{n \rightarrow \infty} V \mu_{n}^{\prime} \quad \text { (vaguely). }
$$

By Corollary 17, $\left(\mu_{n}^{\prime}\right)_{n=1}^{\infty}$ converges vaguely to $\mu_{F}^{\prime}$ as $n \rightarrow \infty$. Let $\left(\omega_{n}^{\prime}\right)_{n=1}^{\infty}$ be another exhaustion of $C F$ and $\mu_{n}^{\prime \prime}$ be the $V$-balayaged measure of $\mu$ on $C \bar{\omega}_{n}^{\prime}$. Then it is easily seen that $\lim _{n \rightarrow \infty} V \mu_{n}^{\prime}=\lim _{n \rightarrow \infty} V \mu_{n}^{\prime \prime}$. By using Corollary 17 again, we have $\mu_{F}^{\prime}=\lim _{n \rightarrow \infty} \mu_{n}^{\prime \prime}$. Thus Lemma 41 is shown.

The above measure $\mu_{F}^{\prime}$ is also called the $V$-balayaged measure of $\mu$ on $F$.

Proposition 42. Let $\left(T_{t}\right)_{t \geqq 0}, V$ and $A$ be the same as in Definition 40 , and let $\Omega$ be an open set in $X$. Assume that $\left(T_{t}\right)_{t \geq 0}$ is weaklyr egular. For $f \in C_{K}(X)$, we put

$$
u_{f}(x)=\int f d \varepsilon_{x, c \Omega}^{\prime} \text { in } X,
$$

where $\varepsilon_{x, C \Omega}^{\prime}$ is the $V$-balayaged measure of $\varepsilon_{x}$ on $C \Omega$. Then $u_{f}$ is $A^{*}$-harmonic in $\Omega$.

Proof. First we shall show that $u_{f}$ is Borel measurable in $X$. By Lemma 41 , it is sufficient to show that, for any open set $\omega$, the function $\int f d \varepsilon_{x, \omega}^{\prime}$ of $x$ is Borel measurable, where $\varepsilon_{x, \omega}^{\prime}$ is the $V$-balayaged measure of $\varepsilon_{x}$ on $\omega$. Let $V^{*} g \in R_{K}\left(V^{*}\right)$. Then $\int|g| d \varepsilon_{x, \omega}^{\prime}<\infty$ and $\int V^{*} g d \varepsilon_{x, \omega}^{\prime}=$ $\int g d V \varepsilon_{x, \omega}^{\prime}$. Since $R_{K}\left(V^{*}\right)$ is dence in $C_{K}(X)$, it suffices to show that, for any $g \in C_{K}^{+}(X)$, the function $\int g d V \varepsilon_{x, \omega}^{\prime}$ of $x$ is Borel measurable. Let $x \in X$ and $\left(x_{n}\right)_{k=1}^{\infty}$ be a sequence $\subset X$ with $\lim _{n \rightarrow \infty} x_{n}=x$. We choose a subsequence $\left(x_{n(k)}\right)_{k=1}^{\infty}$ such that $\varepsilon_{x_{n(k), \omega}}^{\prime}$ converges vaguely and

$$
\varliminf_{n \rightarrow \infty} \int g d V \varepsilon_{x_{n}, \omega}^{\prime}=\lim _{k \rightarrow \infty} \int g d V \varepsilon_{x_{n(k)}, \omega}^{\prime} .
$$

Put $\nu=\lim _{k \rightarrow \infty} \varepsilon_{x_{n(k), \omega}}^{\prime}$. Then supp $(\nu) \subset \bar{\omega}$ and, similarly as in Proposition 23, we have 


$$
V \nu=\lim _{k \rightarrow \infty} V \varepsilon_{x_{n(k), \omega}}^{\prime} \quad \text { (vaguely) }
$$

i.e., $V \nu=V \varepsilon_{x}$ in $\omega$. By the definition of $V$-balayaged measures, we have $V \nu \geqq V \varepsilon_{x, \omega}^{\prime}$, which implies that the function $\int g d V \varepsilon_{x, \omega}^{\prime}$ of $x$ is lower semicontinuous in $X$. Thus we see that $u_{f}$ is Borel measurable in $X$. Let $V \mu \in \mathscr{D}_{K}^{+}(A ; \Omega)$. Choose $h \in \mathbb{C}_{K}^{+}(X)$ such that $V^{*} h(x)>0$ on $\operatorname{supp}(f)$ and that $\int h d V|\mu|<\infty$ (see Lemma 30). Since $R_{K}\left(V^{*}\right)$ is dense in $C_{K}(X)$, there exists a sequence $\left(V^{*} g_{n}\right)_{n=1}^{\infty} \subset R_{K}\left(V^{*}\right)$ such that $\left|f(x)-V^{*} g_{n}(x)\right| \leqq$ $(1 / n) V^{*} h(x)$ on $X$. Then we have

$$
\begin{aligned}
& \left|\int\left(u_{f}(x)-u_{V^{*} g_{n}}(x)\right) d \mu(x)\right| \leqq \frac{1}{n} \int u_{V^{*} h}(x) d|\mu|(x) \\
& \quad \leqq \frac{1}{n} \int V^{*} h(x) d|\mu|(x),
\end{aligned}
$$

where $u_{V^{*} g_{n}}$ and $u_{V^{*} h}$ are defined analogously to $u_{f}$. Consequently, it suffices to show that, for any $V^{*} g \in R_{K}\left(V^{*}\right)$,

$$
\int u_{V^{*} g} d \mu=0 \text {. }
$$

By remarking the first part of this proof, we have

$$
\begin{aligned}
& \int u_{V^{*} g}(x) d \mu(x)=\iint V^{*} g(y) d \varepsilon_{x, C \Omega}^{\prime}(y) d \mu(x) \\
& \quad=\int V^{*} g(y) d\left(\int \varepsilon_{x, C \Omega}^{\prime} d \mu(x)\right)(y)=\int g(y) d V\left(\int \varepsilon_{x, C \Omega}^{\prime} d \mu(x)\right)(y) .
\end{aligned}
$$

Let $\left(\omega_{n}\right)_{n=1}^{\infty}$ be an exhaustion of $\Omega$, and put $\mu_{1}=\mu^{+}, \mu_{2}=\mu^{-}$. We denote by $\mu_{j, n}^{\prime}$ the $V$-balayaged measure of $\mu_{j}$ on $C \bar{\omega}_{n}(j=1,2)$. Then, by virtue of the domination principle for $V$ and by Proposition 16,

$$
V \mu_{j, n+1}^{\prime} \leqq V\left(\int \varepsilon_{x, C \bar{\omega}_{n}}^{\prime} d \mu_{j}(x)\right) \leqq V \mu_{j, n-1}^{\prime}(j=1,2 ; n=2,3, \cdots),
$$

where $\varepsilon_{x, C \bar{\omega}_{n}}^{\prime}$ is the $V$-balayaged measure of $\varepsilon_{x}$ on $C \bar{\omega}_{n}$. This shows that $\int \varepsilon_{x, C \Omega}^{\prime} d \mu_{j}(x)$ is the $V$-balayaged measure of $\mu_{j}$ on $C \Omega(j=1,2)$. Since $V \mu_{1}=V \mu_{2}$ in a certain neighborhood of $C \Omega$, we have

$$
\int \varepsilon_{x, C \Omega}^{\prime} d \mu_{1}(x)=\int \varepsilon_{x, C \Omega}^{\prime} d \mu_{2}(x),
$$


which implies (3.27). This completes the proof.

This implies the following

Corollary 43. Let $\left(T_{t}\right)_{t \geq 0}, V$ and $A$ be the same as above, $\Omega$ be an open set in $X$, and let $g \in C^{+}(X)$ and $f \in C_{K}^{+}(X)$ with $\operatorname{supp}(f) \subset \Omega$. Assume that there exists $\varphi \in \mathscr{D}^{+}\left(V^{*}\right)$ such that $V^{*} \varphi \geqq g$ on $X$. If $g$ is $A^{*}$-superharmonic in $\Omega$ and if $f=-A^{*} g$, i.e., for any $V \mu \in \mathscr{D}_{K}^{+}(A ; \Omega), \int g d \mu=$ $\int f d V \mu$, then

$$
g(x)=\int f d\left(V \varepsilon_{x}-V \varepsilon_{x, C \Omega}^{\prime}\right)+h(x)
$$

on $X$, where $\varepsilon_{x, C \Omega}^{\prime}$ is the same as above and $h$ is an $A^{*}$-harmonic function in $\Omega$. In this case,

$$
h(x)=\int g(y) d \varepsilon_{x, C Q}^{\prime}(y) \text { on } X
$$

Proof. Let $\left(\omega_{n}\right)_{n=1}^{\infty}$ be an exhaustion of $\Omega$ and $\varepsilon_{x, C \bar{\omega}_{n}}^{\prime}$ be the same as above. Then, for any $x \in X$ and any $n \geqq 1, V \varepsilon_{x}-V \varepsilon_{x, C \bar{\omega}_{n}}^{\prime} \in \mathscr{D}_{K}^{+}(A ; \Omega)$. This implies that $g(x) \geqq \int g(y) d \varepsilon_{x, C \Omega}^{\prime}(y)$ on $X$. Let $h$ be the function defined in (3.32). By Proposition 42, $h$ is $A^{*}$-harmonic in $\Omega$. By our assumption, for any $x \in X$ and any $n \geqq 1$,

$$
g(x)-\int g(y) d \varepsilon_{x, C_{\bar{\omega}_{n}}}^{\prime}(y)=\int f d\left(V \varepsilon_{x}-V \varepsilon_{x, \bar{\omega}_{n}}^{\prime}\right) .
$$

Since $\lim _{n \rightarrow \infty} \varepsilon_{x, C \bar{\omega}_{n}}^{\prime}=\varepsilon_{x, C \Omega}^{\prime}$ (vaguely), we have

$$
\begin{aligned}
& \varliminf_{n \rightarrow \infty} \int g d \varepsilon_{x, C \bar{\omega}_{n}}^{\prime} \geqq \int g d \varepsilon_{x, C \Omega}^{\prime} \text { and } \\
& \quad \varliminf_{n \rightarrow \infty} \int\left(V^{*} \varphi-g\right) d \varepsilon_{x, C \bar{\omega}_{n}}^{\prime} \geqq \int\left(V^{*} \varphi-g\right) d \varepsilon_{x, C \Omega}^{\prime} .
\end{aligned}
$$

Remarking that $\left(V \varepsilon_{x, C \bar{\omega}_{n}}^{\prime}\right)_{n=1}^{\infty}$ converges decreasingly to $V \varepsilon_{x, C D}^{\prime}$ as $n \uparrow \infty$, we have

$$
\lim _{n \rightarrow \infty} \int V^{*} \varphi d \varepsilon_{x, C \bar{\omega}_{n}}^{\prime}=\int V^{*} \varphi d \varepsilon_{x, C \Omega}^{\prime}
$$

By combining (3.33), (3.34) and (3.35), we see the required equality. 


\section{$\S 4$. Positive eigen elements for $A$ and completely $A$-superharmonic measures}

We begin by the following

Definition 44. Let $\left(T_{t}\right)_{t \geq 0}$ be a transient diffusion semi-group on $X$ satisfying the condition $\left(C^{*}\right), V$ be the Hunt diffusion kernel for $\left(T_{t}\right)_{t \geq 0}$ and $A$ be the infinitesimal generator of $\left(T_{t}\right)_{t \geqq 0}$.

(1) Given a non-negative number $c$, the set of all non-negative solutions of the equation

$$
-A \mu=c \mu
$$

is denoted by $E(A ; c)$ and called the eigen cone of $c$. Put $E(A)=\cup_{c \geqq 0}$ $E(A ; c)$. We call $\mu \in E(A)$ a non-negative eigen element of $A$.

(2) Given a non-negative number $c$, the set of all non-negative solutions of the equations

$$
\left\{\begin{aligned}
-A \mu & =c \mu \\
\mu & =0 V \text {-n.e. on the boundary of } X
\end{aligned}\right.
$$

is denoted by $E_{0}(A ; c)$ and called the eigen cone of $c$ with zero conditions. Put $E_{0}(A)=\bigcup_{c \geqq 0} E_{0}(A ; c)$. We call $\mu \in E_{0}(A)$ a non-negative eigen element of $A$ with zero conditions.

Now we denote by $H(A)$ the set of all non-negative $A$-harmonic measures in $X$.

Proposition 45. Let $\left(T_{t}\right)_{t \geqq 0}, V, A, E(A ; c)$ and $E_{0}(A ; c)$ be the same as above. Furthermore we assume that $\left(T_{t}\right)_{t \geqq 0}$ is regular. Then, $\mu \in E_{0}(A ; c)$ if and only if

$$
\mu=c V \mu
$$

and we have

$$
E(A ; c)=E_{0}(A ; c) \oplus H(A),
$$

where $\oplus$ denotes the direct sum.

In fact, Remark 29, Theorem 35 and Corollary 39 give the first equivalence, and (4.3) and Theorem 35 give (4.4).

Definition 46. Let $\left(T_{t}\right)_{t \geqq 0}$ be a transient diffusion semi-group on $X$ satisfying the condition $\left(C^{*}\right)$ and $A$ be the infinitesimal generator of $\left(T_{t}\right)_{t \geqq 0}$. A Radon measure $\mu \in M^{+}(X)$ is called a completely $A$-superharmonic 
if, for all $n=0,1,2, \cdots,(-A)^{n} \mu \in \mathscr{D}(A)$ and $(-A)^{n+1} \mu \in M^{+}(X)$, where $(-A)^{0}=I,(-A)^{1}=-A$ and $(-A)^{n+1} \mu=-A\left((-A)^{n} \mu\right)$. In particular, a completely $A$-superharmonic measure $\mu$ is said to be with zero conditions if, for all $n=0,1, \cdots,(-A)^{n} \mu$ vanishes $V$-n.e. on the boundary of $X$, where $V$ is the Hunt diffusion kernel for $\left(T_{t}\right)_{t \geq 0}$.

We denote by $S C(A)$ the set of all completely $A$-superharmonic measures in $X$ and by $S C_{0}(A)$ the set of all completely $A$-superharmonic measures in $X$ with zero conditions.

Evidently $S C(A)$ and $S C_{0}(A)$ are convex cones in $M^{+}(X)$, and $S C(A)$ $\supset E(A)$ and $S C_{0}(A) \supset E_{0}(A)$.

Proposition 47. Let $\left(T_{t}\right)_{t \geqq 0}$ be a transient and regular diffusion semigroup on $X, V$ be the Hunt diffusion kernel for $\left(T_{t}\right)_{t \geqq 0}$ and $A$ be the infinitesimal generator of $\left(T_{t}\right)_{t \geqq 0}$. Assume that, for all $n=1,2, \cdots, V^{n}$ is defined as a diffusion kernel on $X$. Then, for any $\mu \in S C(A)$, we have the following unique representation:

$$
\mu=\sum_{n=0}^{\infty} V^{n} \mu_{n}+\mu_{\infty},
$$

where $\mu_{n} \in H(A)(n=0,1, \cdots)$ and $\mu_{\infty} \in S C_{0}(A)$.

Proof. By Theorem 35, we have inductively, for any $k \geqq 0$ and any $n \geqq k$,

$$
(-A)^{k} \mu=\mu_{k}+V \mu_{k+1}+\cdots+V^{n-k-1} \mu_{n-1}+V^{n-k}\left((-A)^{n} \mu\right),
$$

where $\mu_{k}, \cdots, \mu_{n-1} \in H(A)$. This implies that $\left(V^{n-k}\left((-A)^{n} \mu\right)_{n=k+1}^{\infty}\right.$ is decreasing. Put

$$
\mu_{\infty, k}=\lim _{n \rightarrow \infty} V^{n-k}\left((-A)^{n} \mu\right)
$$

Then we have $\mu_{\infty, 0}=V^{k} \mu_{\infty, k}$. Putting $\mu_{\infty}=\mu_{\infty, 0}$, then $\mu_{\infty} \in S C_{0}(A)$. Putting $k=0$ and letting $n \rightarrow \infty$ in (4.6), we obtain a required representation of $\mu$. By virtue of the unicity of the Riesz decomposition of $(-A)^{k} \mu(k=$ $0,1, \cdots)$, we see the unicity of the representation (4.5) of $\mu$. This completes the proof.

Now we denote by $S(A)$ the set of all non-negative $A$-superharmonic measures in $X$.

Remark 48. Let $\left(T_{t}\right)_{t \geq 0}$ and $A$ be the same as in Proposition 47. Then $S(A)$ is a vaguely closed convex cone in $M^{+}(X)$. 
In fact, let $V$ be the Hunt diffusion kernel for $\left(T_{t}\right)_{t \geqq 0}$. For any $V^{*} f$ $\in R_{K}^{+}\left(V^{*}\right), \operatorname{supp}\left(f^{+}\right) \subset \operatorname{supp}\left(V^{*} f\right)$, and hence, for any vaguely cluster point $\mu$ of $S(A)$, we have $\int f d \mu \geqq 0$. This gives that $\overline{S(A)}=S(A)$.

But, in order to discuss the closedness of $S C(A)$ and that of $E(A)$, we need the following

Definition 49. Let $\left(T_{t}\right)_{t \geqq 0}$ be a transient diffusion semi-group on $X$ satisfying the condition $\left(C^{*}\right)$ and $A$ be the infinitesimal generator of $\left(T_{t}\right)_{t \geqq 0}$. We say that $A$ satisfies the condition $(\mathscr{L})$ if, for any $\left(\mu_{n}\right)_{n=1}^{\infty} \subset S(A)$,

$$
\lim _{n \rightarrow \infty} \mu_{n}=\mu \in S(A) \text { (vaguely) implies } \lim _{n \rightarrow \infty} A \mu_{n}=A \mu \text { (vaguely). }
$$

Proposition 50. Let $\left(T_{t}\right)_{t \geqq 0}$ and $A$ be the same as in Proposition 47. If $A$ satisfies the condition ( $\mathscr{L})$, then, for any constant $c \geqq 0, H(A), E(A ; c)$, $E(A)$ and $S C(A)$ are vaguely closed convex cones in $M^{+}(X)$.

Proof. It is easy to see the vague closedness of $H(A)$ and that of $E(A ; c)$. We remark here $H(A)=E(A ; 0)$. Let $\left(\mu_{n}\right)_{n=1}^{\infty}$ be a sequence in $E(A)$ tending vaguely to $\mu \in M^{+}(X)$ as $n \rightarrow \infty$. Then there exists a sequence of non-negative numbers $\left(c_{n}\right)_{n=1}^{\infty}$ such that $-A \mu_{n}=c_{n} \mu_{n}$. By $E(A) \supset$ $H(A)$, we may assume that $-A \mu \neq 0$. The condition $(\mathscr{L})$ for $A$ gives that $\left(c_{n} \mu_{n}\right)_{n=1}^{\infty}$ converges vaguely to $-A \mu$ as $n \rightarrow \infty$. Hence $\left(c_{n}\right)_{n=1}^{\infty}$ converges to a non-negative number $c$ as $n \rightarrow \infty$, which implies that $\mu \in E(A ; c) \subset E(A)$. Thus we see the vague closedness of $E(A)$. Let $\left(\mu_{n}\right)_{n=1}^{\infty}$ be a sequence of $S C(A)$ tending vaguely to $\mu \in M^{+}(X)$ as $n \rightarrow \infty$. Inductively we have, for any integer $k \geqq 0$,

$$
\lim _{k \rightarrow \infty}(-A)^{k} \mu_{n}=(-A)^{k} \mu \in M^{+}(X) \text { (vaguely), }
$$

which implies that $\mu \in S C(A)$, and hence the vague closedness of $S C(A)$ is shown. This completes the proof.

The above proposition gives the following

Proposition 51. Let $\left(T_{t}\right)_{t \geqq 0}, V$ and $A$ be the same as above. Assume that $A$ satisfies the condition $(\mathscr{L})$ and that, for all $n=1,2, \cdots, V^{n}$ is defined as a diffusion kernel on $X$. Then, for any number $c \geqq 0, S C_{0}(A), E_{0}(A)$ and $E_{0}(A ; c)$ are Borel measurable convex cones in the metrizable space $M^{+}(X)$.

Proof. Since $X$ is with countable basis, $M^{+}(X)$ is metrizable. Choose 
$\left(f_{n}\right)_{n=1}^{\infty} \subset C_{R}^{+}(X)$ such that $\left(f_{n}\right)_{n=1}^{\infty}$ is total in $C_{K}(X)$. For each integer $m \geqq 0$, $n \geqq 1$ and $p \geqq 1$, we put

$$
B_{m, n, p}=\left\{\mu \in S C(A) ; \int f_{n} d \mu_{h, m} \geqq \frac{1}{p}\right\},
$$

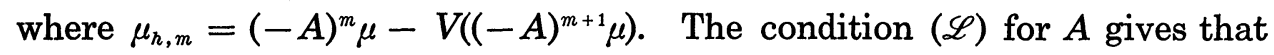
$B_{m, n, p}$ is vaguely closed. Since

$$
S C_{0}(A)=\bigcap_{m=0}^{\infty} \bigcap_{n=1}^{\infty} \bigcap_{p=1}^{\infty}\left(C B_{m, n, p} \cap S C(A)\right),
$$

$S C_{0}(A)$ is Borel measurable. Remarking that $E_{0}(A)=E(A) \cap S C_{0}(A)$ and $E_{0}(A ; c)=E(A ; c) \cap S C_{0}(A)$, we see that $E_{0}(A)$ and $E_{0}(A ; c)$ are Borel measurable. Their convexities are evident, so we achieve the proof.

The following remark shows that the condition $(\mathscr{L})$ for $A$ does not always imply the compactness of the support of $A^{*}$, where $A^{*}$ denotes the dual operator of $A$.

Remark 52. Let $\left(T_{t}\right)_{t \geq 0}$ and $A$ be the same as in Proposition 47 .

(1) If $A^{*}$ is with compact support, i.e., if, for any $V^{*} f \in R_{K}\left(V^{*}\right)$, $\operatorname{supp}(f)$ is compact, then $A$ satisfies the condition $(\mathscr{L})$.

(2) Assume that $\left(T_{t}\right)_{t \geq 0}$ be of convolution type and $A$ satisfies the condition $(\mathscr{L})$. For a positive number $p$, let $A_{p}$ be the infinitesimal generator of the semi-group $\left(T_{p, t}\right)_{t \geq 0}$ defined in (2.43). Then $A_{p}$ also satisfies the condition $(\mathscr{L})$.

In fact, clearly we have (1). We shall show (2). Denote by $\left(V_{p}\right)_{p \geqq 0}$ the resolvent for $\left(T_{t}\right)_{t \geq 0}$. Then, for any $p>0, \mathscr{D}\left(A_{p}\right) \supset M_{K}(X)$ and $A_{p}=$ $p\left(I-p V_{p}\right)$. Let $\left(\mu_{n}\right)_{n=1}^{\infty}$ be a sequence in $S\left(A_{p}\right)$ satisfying $\lim _{n \rightarrow \infty} \mu_{n}=\mu \epsilon$ $S\left(A_{p}\right)$ (vaguely). By Theorem 35 , we have

$$
\begin{aligned}
\mu_{n} & =\left(V+\frac{1}{p} I\right) \nu_{n}+\mu_{n, h}(n=1,2, \cdots) \text { and } \\
\mu & =\left(V+\frac{1}{p} I\right) \nu+\mu_{n},
\end{aligned}
$$

where $\nu_{n}=p\left(I-p V_{p}\right) \mu_{n}, \quad \nu=p\left(I-p V_{p}\right) \mu, \quad \mu_{n, h} \in H\left(A_{p}\right) \quad$ and $\quad \mu_{h} \in H\left(A_{p}\right)$. Since $\mu_{n, h}=p V_{p} \mu_{n, h}$, the resolvent equation gives that, for any $q>0$, $\mu_{n, h}=q V_{q} \mu_{n, h}$, which implies that $\mu_{n, h}$ is invariant with respect to $\left(T_{t}\right)_{t \geq 0}$. Similarly $\mu_{h}$ is also invariant with respect to $\left(T_{t}\right)_{t \geqq 0}$. Since $\left(V \nu_{n}+\mu_{n, h}\right)_{n=1}^{\infty}$ is vaguely bounded, we may assume that it converges vaguely. By Theorem 
35 , its limit is of the form $V \lambda+\mu_{h}^{\prime}$, where $\lambda \in \mathscr{D}^{+}(V)$ and $\mu_{h}^{\prime} \in H(A)$. The condition ( $\mathscr{L})$ for $A$ implies that $\lim _{n \rightarrow \infty} \nu_{n}=\nu$ (vaguely). Hence

$$
\left(V+\frac{1}{p} I\right) \nu+\mu_{h}=\left(V+\frac{1}{p} I\right) \lambda+\mu_{h}^{\prime} .
$$

Since $\left(T_{t}\right)_{t \geqq 0}$ is of convolution type, it is known that $\mu_{h}^{\prime}$ is also invariant with respect to $\left(T_{t}\right)_{t \geqq 0}$ (see [8], p. 343). By virtue of the unicity of the Riesz decomposition of $\mu$, we have $\nu=\lambda$ and $\mu_{h}=\mu_{h}^{\prime}$. Thus (2) is shown.

Hereafter in this paragraph, for any nonzero element $\mu$ of $M^{+}(X)$, we choose a fixed $f_{\mu} \in C^{+}(X)$ such that $f_{\mu}(x)>0$ on $X$ and $\int f_{\mu} d \mu<\infty$. For a transient and regular diffusion semi-group $\left(T_{t}\right)_{t \geq 0}$ on $X$ and its infinitesimal generator $A$, we put, for $\mu \in M^{+}(X)$,

$$
S C(A ; \mu)=\left\{\nu \in S C(A) ; \int f_{\mu} d \nu \leqq 1\right\}
$$

It is easily seen that if $A$ satisfies the condition $(\mathscr{L})$, then $\operatorname{SC}(A ; \mu)$ is vaguely compact convex set in $M^{+}(X)$.

In general, for a convex set $C$ in a locally convex space, we denote by ex $C$ the set of all extreme points of $C$ and, for a convex cone $K$ in a locally convex space, we denote by $\widetilde{\operatorname{exr}} K$ the set of all extreme rays in $K^{6)}$.

Our main theorem is the following

THeOREM 53. Let $\left(T_{t}\right)_{t \geqq t}$ be a transient and regular diffusion semigroup on $X, V$ be the Hunt diffusion kernel for $\left(T_{t}\right)_{t \geqq 0}$ and $A$ be the infinitesimal generator of $\left(T_{t}\right)_{t \geqq 0}$. Assume that, for all integer $n \geqq 1, V^{n}$ is

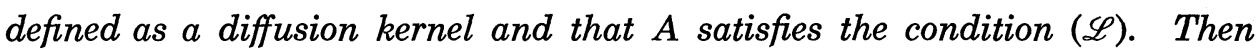
we have:

(1) The set of all extreme rays in $S C(A)$ is represented as follows:

$$
\left.\widetilde{\operatorname{exr}} S C(A)=\left(\bigcup_{n=0}^{\infty} V^{n}(\widetilde{\operatorname{exr}} H(A)) \cap \mathscr{D}\left(V^{n}\right)\right)\right) \cup\left(\bigcup_{t \geqq 0} \widetilde{\operatorname{exr}} E_{0}(A ; t)\right),
$$

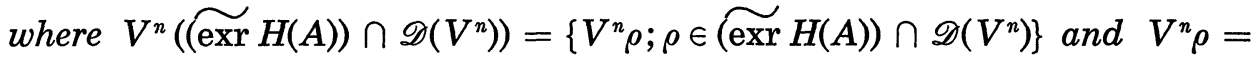
$\left\{\lambda V^{n} \nu ; \lambda \in R^{+}\right\}$with nonzero element $\nu$ of $\rho$, and $S C(A)$ is the closed convex

6) $A$ ray $\rho$ in $K$ is a set of the form $\left\{\lambda x ; \lambda \in R^{+}\right\}$, where $0 \neq x \in K$, and we say that $\rho$ is an extreme ray if, for any $x \in \rho$ and any $y, z \in K, y, z \in \rho$ whenever $x=\lambda y+(1-\lambda) z$ for $\lambda>0$. We denote here by $R^{+}$the totality of all non-negative numbers. 
hull of $\widetilde{\operatorname{exr}} \operatorname{SC}(A)^{7)}$.

(2) For any $\mu \in S C_{0}(A)$, there exists a regular Borel non-negative measure $\Phi$ on $E_{0}(A)$ with $\int d \Phi<\infty$ carried by $\cup_{t \geqq 0} \widetilde{\operatorname{exr}} E_{0}(A ; t)^{8)}$ such that

$$
\left.\mu=\int \lambda d \Phi(\lambda) \text { (i.e., } \int f d \mu=\int\left(\int f d \lambda\right) d \Phi(\lambda) \text { for all } f \in C_{K}(X)\right)
$$

Furthermore, for any $\mu \in S C_{0}(A)$, there exists a Borel non-negative measure $\sigma$ in $(0, \infty)$ with finite total mass and a bounded $\sigma$-measurable mapping $(0, \infty) \ni t \rightarrow \mu_{t} \in E_{0}(A)$ with $\mu_{t} \in E_{0}(A ; t)^{9)}$ such that

$$
\mu=\int_{0}^{\infty} \mu_{t} d \sigma(t)\left(i . e ., \int f d \mu=\int_{0}^{\infty}\left(\int f d \mu_{t}\right) d \sigma(t) \text { for all } f \in C_{K}(X)\right) \text {. }
$$

To prove our main theorem, we use the following three Choquet theorems.

Proposition 54 (see [17], p. 7 and p. 19). Let $C$ be a metrizable compact convex subset of a locally convex space. Then ex $C$ forms a $G_{j}$-set and, for any $x \in C$, there exists a regular Borel probability measure $\mu$ on $C$ carried by ex $C$ which represents $x^{10)}$.

Proposition 55 (see [17], p. 88-89). Let $K$ be a closed convex cone in a locally convex space and suppose that $K$ is union of its caps ${ }^{11)}$. Then $K$ is the closed convex hull of $\widetilde{\operatorname{exr}} K$.

Proposition 56 (see [17], p. 88). Let $K$ be a closed convex cone in a locally convex space and $C$ be its cap. Then every extreme points of $C$ lies on an extreme ray in $K$.

7) In this case, $\widetilde{\operatorname{exr}} S C(A)$ means $\{y \in \rho ; \rho \in \widetilde{\operatorname{exr}} S C(A)\}$ and $\widetilde{\operatorname{exr}} E_{0}(A ; t)$ means the analogous set.

8) We say that a regular Borel measure $\Phi$ on $E_{0}(A)$ is carried by a set $Y \subset E_{0}(A)$ if, there exists a Borel set $B$ such that $B \subset Y$ and $\Phi(C B)=0$.

9) We say that $t \rightarrow \mu_{t}$ is $\sigma$-measurable if, for any $f \in C_{K}(X)$, the function $\int f d \mu_{t}$ of $t$ is $\sigma$-measurable and that is bounded if, for any $f \in C_{K}(X), \int f d \mu_{t}$ is bounded in $(0, \infty)$.

10) $A$ point $x \in C$ is said to be represented by $\mu$ if, for any continuous linear functional $f$,

$$
f(x)=\int f(y) d \mu(y)
$$

11) $A$ non-empty subset $C$ of $K$ is called a cap of $K$ if $C$ is a compact convex subset and if $K-C$ is also convex. 
Proof of Theorem 53. (a) First we shall show that, for any $\mu_{0} \neq 0 \in M^{+}(X)$,

$$
\left(\operatorname{ex} S C\left(A ; \mu_{0}\right)\right) \cap S C_{0}(A) \subset E_{0}(A) .
$$

Let $0 \neq \mu \in S C\left(A ; \mu_{0}\right) \cap S C_{0}(A)$. Theorem 35 and Corollary 39 give that $\mu=V(-A \mu)$. Let $t>0$. Remarking that $T_{t}(-A \mu) \leqq-A \mu$ and $V \cdot T_{t}=$ $T_{t} \cdot V$, we obtain that $T_{t} \mu \in \mathscr{D}^{+}(A)$ and $-A\left(T_{t} \mu\right)=T_{t}(-A \mu)$. Hence we have

$$
(-A)^{n}\left(T_{t} \mu\right)=T_{t}\left((-A)^{n} \mu\right) \in M^{+}(X)(n=0,1, \cdots),
$$

because $\mu=V^{n}\left((-A)^{n} \mu\right)$. This implies that $T_{t} \mu \in S C(A)$. Since $\left(I-T_{t}\right) \mu$ $=\int_{t}^{\infty} T_{s}(-A \mu) d s$, we have also $\left(I-T_{t}\right) \mu \in S C(A)$. Let $0 \neq \mu \in\left(\operatorname{ex} S C\left(A ; \mu_{0}\right)\right)$ $\cap S C_{0}(A)$ and put

$$
c_{1, t}=\int f_{\mu_{0}} d T_{t} \mu \text { and } c_{2, t}=\int f_{\mu_{0}} d\left(I-T_{t}\right) \mu .
$$

Then $c_{j, t}>0(j=1,2)$, because $-A \mu \neq 0$, and $\int f_{\mu_{0}} d \mu=1$. From $T_{t} \mu \in$ $S C\left(A ; \mu_{0}\right),\left(I-T_{t}\right) \mu \in S C\left(A ; \mu_{0}\right)$,

$$
\mu=c_{1, t}\left(\frac{T_{t} \mu}{c_{1, t}}\right)+c_{2, t}\left(\frac{\left(I-T_{t}\right) \mu}{c_{2, t}}\right) \text { and } c_{1, t}+c_{2, t}=1,
$$

it follows that, with a constant $0<c_{t}<1$,

$$
\mu=c_{t} T_{t} \mu,
$$

which implies that, with a constant $a>0$,

$$
-A \mu=\lim _{t \rightarrow 0} \frac{\mu-T_{t} \mu}{t}=\lim _{t \rightarrow 0}\left(\frac{1-c_{t}}{t}\right) \mu=a \mu .
$$

Thus we see (4.18).

(b) Let $0 \neq \mu_{0} \in M^{+}(X)$. We shall show that, for any $\mu \in S C\left(A ; \mu_{0}\right) \cap$ $S C_{0}(A)$, there exists a regular Borel probability measure $\Phi$ on $E_{0}(A)$ carried by (ex $\left.S C\left(A ; \mu_{0}\right)\right) \cap S C_{0}(A)$ such that the analogous equality to (4.16) holds. Put, for each integer $n \geqq 1$,

$$
H_{n}(A)=\left\{V^{n} \mu ; \mu \neq 0 \in \mathscr{D}^{+}\left(V^{n}\right) \cap H(A)\right\}
$$

and $H_{0}(A)=H(A)$. The condition $(\mathscr{L})$ for $A$ implies that, for any $n \geqq 0$, $\oplus_{k=0}^{n} H_{k}(A)$ is vaguely closed and, similarly as in Proposition 51, we see that $H_{n}(A)$ is Borel measurable. Remarking that $\left(H_{n}(A)\right)_{n=1}^{\infty}$ and $S C_{0}(A)-$ $\{0\}$ are mutually disjoint, we have 


$$
\begin{aligned}
& \operatorname{ex} \operatorname{SC}\left(A ; \mu_{0}\right) \\
& \quad=\left(\bigcup_{n=0}^{\infty}\left(\operatorname{ex} S C\left(A ; \mu_{0}\right)\right) \cap H_{n}(A)\right) \cup\left(\left(\operatorname{ex} S C\left(A ; \mu_{0}\right)\right) \cap S C_{0}(A)\right),
\end{aligned}
$$

and $\left(\operatorname{ex} S C\left(A ; \mu_{0}\right)\right) \cap H_{n}(A)(n=0,1, \ldots)$ and $\left(\operatorname{ex} S C\left(A ; \mu_{0}\right)\right) \cap\left(S C_{0}(A)-\{0\}\right)$ are mutually disjoint Borel measurable sets (see Propositions 51 and 54). By Proposition 54, there exists a regular Borel probability measure on $\operatorname{ex} S C\left(A ; \mu_{0}\right)$ such that $\mu=\int \lambda d \Phi(\lambda)$. Put

$$
\Phi_{n}=\left\{\begin{array}{l}
\Phi \text { on }\left(\operatorname{ex} S C\left(A ; \mu_{0}\right)\right) \cap H_{n}(A)(n \geqq 0) \\
0 \text { otherwise }
\end{array} \text { and } \Phi_{\infty}=\Phi-\sum_{n=0}^{\infty} \Phi_{n} .\right.
$$

Then we have

$$
\mu=\sum_{n=0}^{\infty} \int \lambda d \Phi_{n}(\lambda)+\int \lambda d \Phi_{\infty}(\lambda)
$$

By (a), $\Phi_{\infty}$ is a regular Borel non-negative measure on $E_{0}(A)$ carried by $\left(\operatorname{ex} S C\left(A ; \mu_{0}\right)\right) \cap S C_{0}(A)$. For any $n \geqq 0$, the closedness of $\oplus_{k=0}^{n} H_{k}(A)$ implies that $\sum_{k=0}^{n} \int \lambda d \Phi_{k}(\lambda) \in \bigoplus_{k=0}^{n} H_{k}(A)$, and hence Proposition 47 gives that $\int \lambda d \Phi_{n}(\lambda)=0$. Hence we may assume that $\Phi=\Phi_{\infty}$, which gives our assertion.

(c) We shall show that, for any nonzero element $\mu_{0}$ of $M^{+}(X)$,

$$
\left(\operatorname{ex} S C\left(A ; \mu_{0}\right)\right) \cap S C_{0}(A)=\bigcup_{t \geqq 0} \operatorname{ex}\left(E_{0}(A ; t) \cap S C\left(A ; \mu_{0}\right)\right) \text {. }
$$

Evidently we have the inclusion $\subset$, and so we shall show the inverse inclusion. Let $0 \neq \mu \in \operatorname{ex}\left(E_{0}(A ; c) \cap S C\left(A ; \mu_{0}\right)\right)$. Then $c \neq 0$. Assume that, for $\mu_{j} \in S C\left(A ; \mu_{0}\right)(j=1,2), \quad \mu=1 / 2\left(\mu_{1}+\mu_{2}\right)$. Then $\mu_{j} \in S C_{0}(A)(j=1,2)$. By (b), there exists a regular Borel probability measure $\Phi_{j}$ on $E_{0}(A)$ carried by $\left(\operatorname{ex} S C\left(A ; \mu_{0}\right)\right) \cap S C_{0}(A)$ such that $\mu_{j}=\int \lambda d \Phi_{j}(\lambda)(j=1,2)$. By using Propositions 50 and 51 , we see that $E_{0}(A ; c), \bigcup_{c>t \geqq 0} E_{0}(A ; t)$ and $\bigcup_{t>c} E_{0}(A ; t)$ are Borel measurable, because, similarly as in Proposition 50, we see that, for any $s>0, \cup_{t \geqq s} E(A ; t)$ is closed in $M^{+}(X)$ and that $\left(\bigcup_{t \geqq s} E(A ; t)\right) \cap$ $S C_{0}(A)=\bigcup_{t \geqq s} E_{0}(A ; t)$. Put, for $j=1,2$ and $k=0,1,2$,

$$
\begin{gathered}
\Phi_{0, j}=\left\{\begin{array}{l}
\Phi_{j} \text { on } E_{0}(A ; c) \\
0 \text { otherwise, }
\end{array} \quad \Phi_{1, j}=\left\{\begin{array}{l}
\Phi_{j} \text { on }\left(\cup_{c>t \geqq 0} E_{0}(A ; t)\right)-\{0\} \\
0 \text { otherwise, }
\end{array}\right.\right. \\
\Phi_{2, j}=\Phi_{j}-\Phi_{0, j}-\Phi_{1, j} \text { and } \Phi_{k}^{\prime}=\frac{1}{2}\left(\Phi_{k, 1}+\Phi_{k, 2}\right) .
\end{gathered}
$$


For any integer $n \geqq 1$, we have, by the condition $(\mathscr{L})$ for $A$,

$$
\begin{aligned}
\mu=\left(-\frac{1}{c} A\right)^{n} \mu= & \int\left(-\frac{1}{c} A\right)^{n} \lambda d \Phi_{0}^{\prime}(\lambda)+\int\left(-\frac{1}{c} A\right)^{n} \lambda d \Phi_{1}^{\prime}(\lambda) \\
& +\int\left(-\frac{1}{c}\right)^{n} \lambda d \Phi_{2}^{\prime}(\lambda) \\
=\int \lambda d \Phi_{0}^{\prime}(\lambda)+\int & \left(-\frac{c_{\lambda}}{c}\right)^{n} \lambda d \Phi_{1}^{\prime}(\lambda)+\int\left(-\frac{c_{\lambda}}{c}\right)^{n} \lambda d \Phi_{2}^{\prime}(\lambda),
\end{aligned}
$$

where $c_{\lambda}$ is a positive constant satisfying $-A \lambda=c_{\lambda} \lambda$. We remark here that the mapping $\left(E_{0}(A)-\{0\}\right) \ni \lambda \rightarrow c_{\lambda}$ is continuous. By letting $n \rightarrow \infty$ in (4.30), we see that $\mu=\int \lambda d \Phi_{0}^{\prime}(\lambda)$. This implies that $\mu_{j}=\int \lambda d \Phi_{0, j}(\lambda)$ $(j=1,2)$. Since $\int f_{\mu_{0}} d \mu=1$, we have $\mu=\mu_{j}(j=1,2)$, Thus we see that (4.28) holds.

(d) Since $S C(A)=\bigcup_{0 \neq \mu \in S C(A)} S C(A ; \mu)$, Proposition 55 gives that $S C(A)$ is the closed convex hull of $\widetilde{\operatorname{exr}} \operatorname{SC}(A)$. Evidently we have

$$
\left.\widetilde{\operatorname{exr}} S C(A) \subset\left(\bigcup_{n=0}^{\infty} V^{n}(\widetilde{\operatorname{exr}} H(A)) \cap \mathscr{D}\left(V^{n}\right)\right)\right) \cup\left(\bigcup_{t \geqq 0} \widetilde{\operatorname{exr}} E_{0}(A ; t)\right)
$$

and

$$
\left.\widetilde{\operatorname{exr}} S C(A) \supset \bigcup_{n=0}^{\infty} V^{n}(\widetilde{\operatorname{exr}} H(A)) \cap \mathscr{D}\left(V^{n}\right)\right)
$$

by Proposition 47. Let $t>0$ and $\rho \in \widetilde{\operatorname{exr}} E_{0}(A ; t)$. We choose a nonzero element $\mu$ of $\rho$. Then $\mu \in \operatorname{ex}\left(E_{0}(A ; t) \cap S C(A ; \mu)\right)$, and hence (c) implies that $\mu \in(\operatorname{ex} S C(A ; \mu)) \cap S C_{0}(A)$. By Proposition 56, we have $\rho \in \widetilde{\operatorname{exr}} S C(A)$. This implies that (4.15) holds. Proposition 56, (b) and (c) give also (4.16).

(e) Finally, we shall show (4.17). Let $\mu \in S C_{0}(A)$ and $\Phi$ be a regular Borel non-negative measure with $\int d \Phi<\infty$ defined by (4.16). By (b) and (c), $\Phi$ is carried by $(\operatorname{ex} S C(A ; \mu)) \cap\left(\bigcup_{t \geqq 0} E_{0}(A ; t)\right)$. For any $t>0$, we put

$$
\Phi_{t}=\left\{\begin{array}{l}
\Phi \text { on } \bigcup_{t \geqq s \geqq 0} E_{0}(A ; s) \\
0 \text { otherwise }
\end{array} \text { and } v(t)=\int d \Phi_{t}=\int\left(\int f_{\mu} d \lambda\right) d \Phi_{t}(\lambda),\right.
$$

because $\int f_{\mu} d \lambda=1$ for any nonzero element $\lambda$ of $\operatorname{ex} S C(A ; \mu)$. Then $v(t)$ is a bounded non-negative increasing function on $(0, \infty)$. Let $\sigma$ be a non- 
negative Borel measure in $(0, \infty)$ such that $v(t)=\int_{0}^{t} d \sigma$. Then $\int_{0}^{\infty} d \sigma<\infty$. For $f \in C_{K}(X)$, we put

$$
v_{f}(t)=\int\left(\int f d \lambda\right) d \Phi_{t}(\lambda)
$$

Then there exists a real Borel measure $\sigma_{f}$ in $(0, \infty)$ such that $v_{f}(t)=\int_{0}^{t} d \sigma_{f}$. We have also $\int_{0}^{\infty} d\left|\sigma_{f}\right|<\infty$. Since $|f| \leqq c_{f} f_{\mu}$ on $X$ for some positive number $c_{f}$, we have, for any $t>s>0$,

$$
\left|v_{f}(t)-v_{f}(s)\right| \leqq c_{f}(v(t)-v(s)),
$$

which shows that $\sigma_{f}$ is absolutely continuous with respect to $\sigma$. By the Radon-Nikodym theorem, there exists a $\sigma$-integrable function $\tilde{f}$ on $(0, \infty)$ such that $d \sigma_{f}=\tilde{f} d \sigma$. We have also $|\tilde{f}| \leqq c_{f} \sigma$-a.e.. By (4.32), we have, for any $f, g \in C_{K}^{+}(X)$, and any constants $a, b$,

$$
\widetilde{a f+b g}=a \tilde{f}+b \tilde{g} \sigma \text {-a.e.. }
$$

We choose a countable set of continuous functions $\left(f_{n}\right)_{n=1}^{\infty} \subset C_{K}^{+}(X)$ such that $\left(f_{n}\right)_{n=1}^{\infty}$ is total in $C_{K}(X)$. By (4.34), there exists a Borel set $F$ in $(0, \infty)$ such that $\sigma(C F)=0$ and that, for any $t \in F$, any rational number $r$ and any integers $n \geqq 1$ and $m \geqq 1$,

$$
\begin{aligned}
&\left(r \tilde{f}_{n}\right)(t)=t \tilde{f}_{n}(t), \lim _{\delta \downarrow 0} \frac{1}{\delta} \int_{t}^{t+\delta} \tilde{f}_{n} d \sigma=\tilde{f}_{n}(t) \quad \text { and } \\
&\left(\widetilde{f_{n}+f_{m}}\right)(t)=\tilde{f}_{n}(t)+\tilde{f}_{m}(t) .
\end{aligned}
$$

For any $t \in C F$, the mapping $f_{n} \rightarrow \tilde{f}_{n}(t)$ can be extended to a positive linear form on $C_{K}(X)$ in the usual way, and hence there exists a uniquely determined non-negative Radon measure $\mu_{t}$ in $X$ such that $\tilde{f}_{n}(t)=\int f_{n} d \mu_{t}$ for all $n \geqq 1$. By defining $\mu_{t}=0$ for all $t \in C F$, we see that $(0, \infty) \ni t \rightarrow$ $\mu_{t} \in M^{+}(X)$ is $\sigma$-measurable. Since $\int f_{\mu} d \mu_{t} \leqq 1$ for all $t \in(0, \infty),(0, \infty) \ni t \rightarrow$ $\mu_{t} \in M^{+}(X)$ is bounded. Furthermore we have

$$
\mu=\int_{0}^{\infty} \mu_{t} d \sigma(t)
$$

The condition $(\mathscr{L})$ for $A$ and the second equality in (4.35) give that 
$\mu_{t} \in E(A ; t)$ for all $t \in(0, \infty)$. By Theorem 35 and (4.36), we may assume that $\mu_{t} \in E_{0}(A ; t)$. This completes the proof.

Now we notice the following equality:

$$
\begin{array}{r}
S C_{0}(A)=\left\{\int_{0}^{\infty} \mu_{t} d \sigma(t) ; \sigma \in M_{b}^{+}((0, \infty)), t \rightarrow \mu_{t} \in E_{0}(A ; t):\right. \text { bounded and } \\
\sigma \text {-measurable }\},
\end{array}
$$

where $M_{b}^{+}((0, \infty))$ denotes the totality of all non-negative Borel measures in $(0, \infty)$ with finite total mass. In fact, let $\sigma \in M_{b}^{+}((0, \infty))$ and $(0, \infty) \ni t$ $\rightarrow \mu_{t} \in E_{0}(A ; t)$ be a bounded $\sigma$-measurable mapping. Put $\sigma_{n}=\sigma$ on $[1 / n, n]$ and $\sigma_{n}=0$ otherwise $(n=1,2, \cdots)$. Then the condition $(\mathscr{L})$ for $A$ gives that, for all $n=1,2, \cdots$ and $m=0,1,2, \cdots$,

$$
\begin{aligned}
(-A)^{m} \int_{0}^{\infty} \mu_{t} d \sigma_{n}(t) & =\int_{0}^{\infty} t^{m} \mu_{t} d \sigma_{n}(t) \text { and } \\
\int_{0}^{\infty} \mu_{t} d \sigma_{n}(t) & =V^{m}\left(\int_{0}^{\infty} t^{m} \mu_{t} d \sigma_{n}(t)\right) .
\end{aligned}
$$

By letting $n \rightarrow \infty$ in (4.38) and using the condition $(\mathscr{L})$ for $A$, we have, for any $m \geqq 0, \int_{0}^{\infty} t^{m} \mu_{t} d \sigma(t) \in M^{+}(X)$ and

$$
\begin{aligned}
(-A)^{m} \int_{0}^{\infty} \mu_{t} d \sigma(t) & =\int_{0}^{\infty} t^{m} \mu_{t} d \sigma(t) \quad \text { and } \\
\int_{0}^{\infty} \mu_{t} d \sigma(t) & =V^{m}\left(\int_{0}^{\infty} t^{m} \mu_{t} d \sigma(t)\right) .
\end{aligned}
$$

By combining Theorem 53 and (4.39), we have (4.37).

For $\mu \in M(X)$, we write $\rho(\mu)=\left\{c \mu ; c \in R^{+}\right\}$. In particular, we have the following

Proposition 57. Let $X$ be a locally compact abelian group with countable basis and $\xi$ be a Haar measure on $X$. Let $\left(T_{t}\right)_{t \geq 0}$ be a transient diffusion semi-group of convolution type on $X$ and $\alpha_{t}$ be the non-negative Radon measure on $X$ defining $T_{t}$ (see (2.17)). Assume that the infinitesimal generator $A$ of $\left(T_{t}\right)_{t \geqq 0}$ satisfies the condition $(\mathscr{L})$ and let $\operatorname{Exp}(X)$ be the totality of all positive continuous exponential functions on $X^{12)}$. Then we have:

12) $A$ real-valued function $\varphi$ on $X$ is said to be exponential if, for any $x, y \in X$, $\varphi(x+y)=\varphi(x) \cdot \varphi(y)$. 
(1) $\widetilde{\operatorname{exr}} H(A) \subset\left\{\rho(\varphi \xi) ; \varphi \in \operatorname{Exp}(X), \int \varphi d \alpha_{t}=1\right.$ for all $\left.t \geqq 0\right\} \subset H(A)^{13)}$

(2) For any $c>0, \widetilde{\operatorname{exr}} E_{0}(A ; c) \subset\left\{\rho(\varphi \xi) ; \varphi \in \operatorname{Exp}(X), c \int_{0}^{\infty}\left(\int \varphi d \alpha_{t}\right) d t=1\right\}$ $\subset E_{0}(A ; c)$.

Proof. It is known that

$$
\begin{aligned}
H(A) & =\left\{\mu \in M^{+}(X) ; \mu=\mu * \alpha_{t} \text { for all } t \geqq 0\right\} \\
& =\left\{\mu \in M^{+}(X) ; \mu=\mu * \alpha_{t_{0}} \text { for some } t_{0}>0\right\}
\end{aligned}
$$

(see [8], p. 343). This implies the second inclusion in (1). By the ChoquetDeny theorem (see [5]) ${ }^{13}$, we see the first inclusion in (1). Similarly we see the assertion (2). Lastly in this paragraph, we shall discuss the Bernstein theorem. Put

(4.41) $T_{t}: M_{K}((0, \infty)) \ni \mu \rightarrow$ the restriction of $\tau_{-t} \mu$ to $(0, \infty) \in M((0, \infty))$

for all $t \geqq 0$, where $\tau_{-t}$ is the translation of $-t$. Then $\left(T_{t}\right)_{t \geq 0}$ is transient and regular diffusion semi-group on $(0, \infty)$, and its infinitesimal generator $A$ is equal to $d / d t$. Denote by $d t$ the Lebesgue measure in $(0, \infty)$. Since the Hunt diffusion kernel $V$ for $\left(T_{t}\right)_{t \geq 0}$ satisfies

$$
V \mu=\left(\int_{t}^{\infty} d \mu\right) d t \text { for all } \mu \in M_{K}((0, \infty))
$$

and

$$
H\left(\frac{d}{d t}\right)=\rho(d t) \text { and } E_{0}\left(\frac{d}{d t} ; c\right)=\rho(\exp (-c t) d t) \text { for all } c>0 .
$$

Hence, our main theorem implies the Bernstein theorem. We remark here that

$$
\begin{aligned}
& V^{n} \mu=\left(\int_{t}^{\infty} \frac{1}{(n-1) !}(x-t)^{n-1} d \mu(x)\right) d t \\
& \quad \text { for all } \mu \in M_{K}((0, \infty)) \text { and } n=1,2, \cdots,
\end{aligned}
$$

and that

13) This shows that, for a non-negative Radon measure $\sigma$ in $X$, the solution $\mu$ of the convolution equation $\mu=\mu * \sigma$ is of form

$$
\mu=\left(\int \varphi d \lambda(\varphi)\right) \xi,
$$

where $\lambda$ is a regular Borel measure with finite total mass on $\left\{\varphi \in \operatorname{Exp}(X) ; \int \varphi d \sigma=1\right\}$. 


$$
d t \notin \mathscr{D}^{+}\left(V^{n}\right) \text { for all } n=1,2, \cdots
$$

\section{§5. Application to elliptic differential operators}

In this paragraph, we consider the same setting as in S. Itô's paper [10]. Let $D$ be a subdomain of an orientable $N$-dimensional $C^{\infty}$-manifold $(N \geqq 2)$ and $L$ be an elliptic differential operator of the form:

$$
\begin{aligned}
L u(x)= & \sum_{i, j=1}^{N} \frac{1}{\sqrt{a(x)}} \frac{\partial}{\partial x^{i}}\left(\sqrt{a(x)} \cdot a^{i j}(x) \frac{\partial u}{\partial x^{j}}(x)\right) \\
& +\sum_{i=1}^{N} b^{i}(x) \frac{\partial u}{\partial x^{i}}(x)+c(x) u(x)
\end{aligned}
$$

for $u \in C^{2}(D)^{14)}$ and $x=\left(x^{1}, \cdots, x^{N}\right) \in D$, where $\left(a^{i j}(x)\right)_{i, j=1}^{N}$ is a contravariant tensor of class $C^{\infty}$ in $D$ and is symmetric and strictly positivedefinite for each $x \in D, a(x)=\operatorname{det}\left(a_{i j}(x)\right)=\operatorname{det}\left(a^{i j}(x)\right)^{-1},\left(b^{i}(x)\right)_{i=1}^{N}$ is a contravariant vector of class $C^{\infty}$ in $D$ and $c(x)$ is a non-positive function of class $C^{\infty}$ in $D$. We shall denote by $d x$ the volume element with respect to the Riemannian metric defined by the tensor $\left(a_{i j}(x)\right)_{i, j=1}^{N}$. The formally adjoint operator $L^{*}$ of $L$ is defined by

$$
\begin{aligned}
L^{*} v(x)= & \sum_{i, j=1}^{N} \frac{1}{\sqrt{a(x)}} \frac{\partial}{\partial x^{i}}\left(\sqrt{a(x)} \cdot a^{i j}(x) \frac{\partial v}{\partial x^{j}}(x)\right) \\
& -\sum_{i=1}^{N} \frac{1}{\sqrt{a(x)}} \frac{\partial}{\partial x^{i}}\left(\sqrt{a(x)} \cdot b^{i}(x) \cdot v(x)\right)+c(x) v(x)
\end{aligned}
$$

for $v \in C^{2}(D)$.

Evidently we have the following

Remark 58. Let $u$ and $v$ be in $C^{2}(D)$. If $u \in C_{K}^{2}(D)$ or $v \in C_{K}^{2}(D)$, then we have

$$
\int L u(x) v(x) d x=\int u(x) L^{*} v(x) d x .
$$

Definition 59 (see [10]). Let $\Omega$ be a subdomain of $D$. We say that $\Omega$ satisfies the condition $(S)$ if its closure $\bar{\Omega}$ is contained in $D$ and its boundary $\partial \Omega$ consists of finite number of simple closed hypersurfaces of class $C^{3}$.

Proposition 60 (see [9], Theorem 1). Let $\Omega$ be a subdomain of $D$

14) We denote by $C^{n}(D)=\left\{f \in C(D)\right.$; $f$ is of class $C^{n}$ in $\left.D\right\}$ for $n \geqq 1$ and by $C^{\infty}(D)$ $=\cap_{n=1}^{\infty} C^{n}(D)$. We write also $C_{K}^{n}(D)=C^{n}(D) \cap C_{K}(D)$ and $C_{K}^{\infty}(D)=C^{\infty}(D) \cap C_{K}(D)$. 
satisfying the condition (S). Then there exists one and only one fundamental solution $U_{\Omega}(t, x, y)$ of the initial-boundary value problem:

Given $u_{0} \in C(\bar{\Omega})$ and $\varphi \in C((0, \infty) \times \partial \Omega)$,

$$
\left\{\begin{array}{l}
\frac{\partial u}{\partial t}(t, x)=L u(t, x) \text { for each }(t, x) \in(0, \infty) \times \Omega \\
u(0, x)=u_{0}(x) \text { for each } x \in \bar{\Omega} \\
u(t, x)=\varphi(t, x) \text { for each }(t, x) \in(0, \infty) \times \partial \Omega .
\end{array}\right.
$$

Furthermore $U_{\Omega}(t, x, y)$ satisfies the following five conditions:

(5.5) $U_{\Omega}(t, x, y)$ is a non-negative finite continuous function on $(0, \infty) \times$ $\bar{\Omega} \times \bar{\Omega}$ and $U_{\Omega}(t, x, y)=0$ if and only if $x \in \partial \Omega$ or $y \in \partial \Omega$.

(5.7) $\int U_{\Omega}(t, x, y) U_{\Omega}(s, y, z) d y=U_{\Omega}(t+s, x, z)$ for any $t>0, s>0$ and any $(x, z) \in \bar{\Omega} \times \bar{\Omega}$.

(5.8) For any $u_{0} \in C(\bar{\Omega})$, we put $u(t, x)=\int U_{\Omega}(t, x, y) u_{0}(y) d y$. Then $u(t, x)$ is the unique solution of (5.4) with $\varphi=0$.

(5.9) For any $u_{0} \in C(\bar{\Omega})$, we put $u^{*}(t, x)=\int U_{\Omega}(t, y, x) u_{0}(y) d y$. Then $u^{*}(t, x)$ is the unique solution of the initial-boundary value problem:

$$
\left\{\begin{array}{l}
\frac{\partial u}{\partial t}(t, x)=L^{*} u(t, x) \text { for each }(t, x) \in(0, \infty) \times \Omega \\
u(0, x)=u_{0}(x) \text { for each } x \in \bar{\Omega} \\
u(t, x)=0 \text { for each }(t, x) \in(0, \infty) \times \partial \Omega
\end{array}\right.
$$

The following remark is elementary.

Remark 61. Let $\Omega$ be a subdomain of $D$. Then there exists a sequence $\left(\Omega_{n}\right)_{n=1}^{\infty}$ of subdomains in $\Omega$ satisfying the condition $(S)$ such that $\bar{\Omega}_{n} \subset$ $\Omega_{n+1}, \bigcup_{n=1}^{\infty} \Omega_{n}=\Omega$.

We call $\left(\Omega_{n}\right)_{n=1}^{\infty}$ a regular exhaustion of $\Omega$.

Proposition 62 (see [9], Lemma 5.4). Let $\Omega$ and $\left(\Omega_{n}\right)_{n=1}^{\infty}$ be the same as above. Then $\left(U_{\Omega_{n}}(t, x, y)\right)_{n=1}^{\infty}$ converges increasingly to a continuous func- 
tion $U_{\Omega}(t, x, y)$ in $(0, \infty) \times \Omega \times \Omega^{15)}$.

We remark here that $U_{\Omega_{n}}(t, x, y) \rightarrow U_{\Omega}(t, x, y)$ in $C((0, \infty) \times \Omega \times \Omega)$ as $n \rightarrow \infty$ and that $U_{\Omega}(t, x, y)$ does not depend on the choice of $\left(\Omega_{n}\right)_{n=1}^{\infty}$.

Corollary 63. Let $\Omega$ and $U_{\Omega}(t, x, y)$ be the same as above. Then we have:

(1) For $t>0, s>0$ and $(x, z) \in \Omega \times \Omega$,

$$
\int U_{\Omega}(t, x, y) U_{\Omega}(s, y, z) d y=U_{\Omega}(t+s, x, z) .
$$

(2) For any $f \in C_{K}(\Omega)$, we put

$$
u(t, x)=\left\{\begin{array}{l}
\int U_{\Omega}(t, x, y) f(y) d y \text { in }(0, \infty) \times \Omega \\
f(x) \text { on }\{0\} \times \Omega
\end{array}\right.
$$

and

$$
u^{*}(t, x)=\left\{\begin{array}{l}
\int U_{\Omega}(t, y, x) f(y) d y \text { in }(0, \infty) \times \Omega \\
f(x) \text { on }\{0\} \times \Omega .
\end{array}\right.
$$

Then $u(t, x)$ and $u^{*}(t, x)$ are finite continuous in $[0, \infty) \times \Omega$.

Proof. Since $U_{\Omega_{n}}(t, x, y) \uparrow U_{\Omega}(t, x, y)$ as $n \uparrow \infty$, (5.7) gives (5.11). To show (2), we may assume that $f$ is non-negative. Put

$$
u_{n}(t, x)=\left\{\begin{array}{l}
\int U_{\Omega_{n}}(t, x, y) f(y) d y \text { in }(0, \infty) \times \Omega \\
f(x) \text { on }\{0\} \times \Omega .
\end{array}\right.
$$

Then $u_{n}$ is finite continuous on $[0, \infty) \times \Omega$. Since $\left(u_{n}(t, x)\right)_{n=1}^{\infty}$ converges increasingly to $u(t, x)$ as $n \rightarrow \infty, u$ is lower semi-continuous on $[0, \infty) \times \Omega$. Evidently $u(t, x)$ is finite continuous in $(0, \infty) \times \Omega$. Let $t_{0}$ be a fixed positive number. Then there exists a constant $c>0$ such that $c \int U_{\Omega}\left(t_{0}, x, y\right) f(y) d y \geqq f(x)$ on $\Omega$. Hence $c u\left(t_{0}+t, x\right)-u(t, x)$ is also lower semi-continuous on $[0, \infty) \times \Omega$. This implies that $u(t, x)$ is finite continuous on $[0, \infty) \times \Omega$. By the similar argument, we see that $u^{*}(t, x)$ is also finite continuous on $[0, \infty) \times \Omega$. This completes the proof.

15) We may assume that $U_{\Omega_{n}}(t, x, y)$ is a finite continuous function in $(0, \infty) \times \Omega \times \Omega$, by defining that $U_{\Omega_{n}}(t, x, y)=0$ if $x \in C \Omega_{n}$ or $y \in C \Omega_{n}$. 
Let $\Omega$ be a subdomain of $D$. For any $t>0$, we define linear operators $T_{L, \Omega, t}$ and $T_{L^{*}, \Omega, t}$ from $M_{K}(\Omega)$ into $M(\Omega)$ as follows:

$$
T_{L, Q, t} \mu=\left(\int U_{\Omega}(t, x, y) d \mu(y)\right) d x \text { and } T_{L^{*}, \Omega, t} \mu=\left(\int U_{\Omega}(t, y, x) d \mu(y)\right) d x
$$

By Corollary 63, we have the following

Remark 64. Putting $T_{L, Q, 0}=T_{L^{*}, \Omega, 0}=I$, we see that $\left(T_{L, Q, t}\right)_{t \geqq 0}$ and $\left(T_{L^{*}, Q, t}\right)_{t \geq 0}$ are diffusion semi-groups on $\Omega$.

For the sake of simplicity, we write $T_{L, t}=T_{L, D, t}$ and $T_{L^{*}, t}=T_{L^{*}, D, t}$ $(t \geqq 0)$.

Proposition 65. The diffusion semi-group $\left(T_{L, t}\right)_{t \geq 0}$ on $D$ is transient if and only if the Green function $G(x, y)$ of $L$ on $D^{18)}$ exists. If $G(x, y)$. exists, then $G(x, y)=\int_{0}^{\infty} U_{D}(t, x, y) d t$.

This follows from the following

Proposition 66. The Green function $G(x, y)$ of $L$ on $D$ exists if and only if there exists a non-constant lower semi-continuous and locally integrable function $f$ satisfying $0 \leqq f \leqq \infty, f \not \equiv \infty$ and $-L f \geqq 0$ in the sense of distributions in D. Furthermore, if $G(x, y)$ exists, we have $G(x, y)=$ $\int_{0}^{\infty} U_{D}(t, x, y) d t$. For any $y \in D$, the functions $G(x, y)$ and $G(y, x)$ of $x$ belong to $C^{\infty}(D-\{y\})$, and for any $f \in C_{K}^{\infty}(D), G f(x)=\int G(x, y) f(y) d y \in C^{\infty}(D)$ and

$$
L G f=G(L f)=-f .
$$

S. Itô shows the above assertion in the case of $c(x) \equiv 0$ (see [10]). In the case that $c(x) \not \equiv 0$, we see, in the same manner as in [10], that there exists the Green function of $L$ on $D$ (see also [9] and [12]).

Remark 67 (see [9], $\S 10$ and [10]). If $G(x, y)$ exists, then $G^{*}(x, y)=$ $G(y, x)=\int_{0}^{\infty} U_{D}(t, y, x) d t$ is the Green function of $L^{*}$ on $D$ and, for any

16) For an open set $\Omega$ in $D$, the Green function $G_{\Omega}(x, y)$ of $L$ on $\Omega$ means a nonnegative continuous function in $\Omega \times \Omega$ in the extended sense satisfying the following conditions :

(a) $G_{\Omega}(x, y)<\infty$ if $x \neq y$.

(b) $L_{x} G_{\Omega}(x, y)=-\varepsilon_{y}$ in the sense of distributions.

(c) For any $y \in \Omega$ and any non-negative function $h \in C^{2}(\Omega)$ with $L h=0$ in $\Omega$, $G_{\varrho}(x, y) \geqq h(x)$ in $\Omega$ implies $h \equiv 0$. 
$f \in C_{K}^{\infty}(D), G^{*} f(x)=\int G^{*}(x, y) f(y) d y \in C^{\infty}(D)$ and

$$
L^{*} G^{*} f=G^{*}\left(L^{*} f\right)=-f .
$$

Proof of Proposition 65. We remark that, if $\left(T_{L, t}\right)_{t \geqq 0}$ is transient, then, for any nonzero element $\mu$ of $M_{K}^{+}(D), \int_{0}^{\infty} \int U_{D}(t, x, y) d \mu(y) d t$ is a non-constant lower semi-continuous and locally integrable function in $D$ satisfying $-L\left(\int_{0}^{\infty} \int U_{D}(t, x, y) d \mu(y) d t\right) \geqq 0$ in the sense of distributions in $D$. If $G(x, y)$ exists, Proposition 66 and Remark 67 give that, for any $f \in C_{K}^{+}(D)$, $\int_{0}^{\infty} T_{L, t}^{*} f d t$ is a non-negative lower semi-continuous function in $D$ and that, for any $f \in C_{K}^{\infty}(D), \int_{0}^{\infty} T_{L, t}^{*} f d t=G^{*} f \in C^{\infty}(D)$, and hence $\left(T_{L, t}\right)_{t \geq 0}$ is transient.

Hereafter, we shall always assume that the Green function $G(x, y)$ of $L$ on $D$ exists. Define the linear operators $V_{L}$ and $V_{L^{*}}$ from $M_{K}(D)$ into $M(D)$ as follows:

$$
V_{L} \mu=(G \mu) d x \text { and } V_{L^{*}} \mu=\left(G^{*} \mu\right) d x,
$$

where $G \mu(x)=\int G(x, y) d \mu(y)$ and $G^{*} \mu(x)=\int G^{*}(x, y) d \mu(y)$. Then $V_{L}$ and $V_{L^{*}}$ respectively are the Hunt diffusion kernel for $\left(T_{L, t}\right)_{t \geqq 0}$ and that for $\left(T_{L^{*}, t}\right)_{t \geqq 0}$.

Remark 68. Let $\mu \in M_{K}(D)$. Then

$$
L G \mu=-\mu \text { and } L^{*} G^{*} \mu=-\mu
$$

in the sense of distributions in $D$.

In fact, $V_{L}$ and $V_{L^{*}}$ are defined, so that $G \mu$ and $G^{*} \mu$ are locally integrable. The two equalities in (5.19) follow from (5.16) and (5.17). The two equalities (5.16) and (5.17) imply also the following

Remark 69. We have $R_{K}\left(V_{L}^{*}\right) \supset C_{K}^{\infty}(D)$ and $R_{K}\left(V_{L^{*}}^{*}\right) \supset C_{K}^{\infty}(D)$, i.e., $\left(T_{L, t}\right)_{t \geq 0}$ and $\left(T_{L^{*}, t}\right)_{t \geqq 0}$ satisfy the condition $\left(C^{*}\right)$. Let $A_{L}$ and $A_{L^{*}}$ be the infinitesimal generator of $\left(T_{L, t}\right)_{t \geqq 0}$ and that of $\left(T_{L^{*}, t}\right)_{t \geqq 0}$, respectively. Then, for any $\mu \in \mathscr{D}\left(A_{L}\right)$ (resp. $\mu \in \mathscr{D}\left(A_{L^{*}}\right)$ ),

$$
A_{L} \mu=L \mu\left(\text { resp. } A_{L^{*} \mu}=L^{*} \mu\right)
$$

in the sense of distributions. 
Let $\Omega$ be a subdomain of $D$ satisfying the condition $(S)$. It is wellknown that, for any $y \in \Omega$, there exists the $V_{L}$-balayaged measure $\varepsilon_{y, c \Omega}^{\prime}$ (resp. $V_{L^{*}}$-balayaged measure $\left.\varepsilon_{y, C \Omega}^{\prime \prime}\right)$ of $\varepsilon_{y}$ on $C \Omega$. We have $\operatorname{supp}\left(\varepsilon_{y, C \Omega}^{\prime}\right) \subset \partial \Omega$, $\operatorname{supp}\left(\varepsilon_{y, C \Omega}^{\prime \prime}\right) \subset \partial \Omega$,

$$
\begin{gathered}
\int_{0}^{\infty} U_{\Omega}(t, x, y) d t=G(x, y)-G \varepsilon_{y, c \Omega}^{\prime}(x) \quad \text { and } \\
\int_{0}^{\infty} U_{\Omega}(t, y, x) d t=G^{*}(x, y)-G \varepsilon_{y, C \Omega}^{\prime \prime}(x)
\end{gathered}
$$

(see, for example, [11], p. 333). Put $G_{\Omega}(x, y)=\int_{0}^{\infty} U_{\Omega}(t, x, y) d t$. Then $G_{\Omega}(x, y)$ is the Green function of $L$ on $\Omega$. In this case,

$$
\lim _{y \rightarrow \Omega} G_{\Omega}(x, y)=\lim _{y \rightarrow \Omega} G_{\Omega}(y, x)=0 \text { for all } x \in \Omega .
$$

To apply our main theorem to $L$, we need the following

THEOREM 70. Two diffusion semi-groups $\left(T_{L, t}\right)_{t \geq 0}$ and $\left(T_{L^{*}, t}\right)_{t \geq 0}$ are regular.

Proof. We shall show only that $\left(T_{L, t}\right)_{t \geq 0}$ is regular, because the other is proved similarly. By Remark 69, it suffices to show that $\left(T_{L, t}\right)_{t \geq 0}$ satisfies the condition $\left(D^{*}\right)$. By Proposition 62, Remark 61 and (5.21), $\left(T_{L, t}\right)_{t \geq 0}$ is weakly regular. Let $\left(D_{n}\right)_{n=1}^{\infty}$ be a regular exhaustion of $D$ and put $T_{n, t}$ $=T_{L, D_{n}, t}(t \geqq 0 ; n=1,2, \cdots)$. Since, for any $\mu \in M_{K}^{+}(D), T_{n, t} \mu \leqq T_{L, t} \mu$ in $D_{n},\left(T_{n, t}\right)_{t \geq 0}$ is also a transient and weakly regular diffusion semi-group on $D_{n}$. Let $V_{L, n}$ the Hunt diffusion kernel for $\left(T_{n, t}\right)_{t \geq 0}$. Then $V_{L, n} \mu=$ $\left(G_{D_{n}} \mu\right) d x$ for any $n \geqq 1$. First we shall show that if, for any $n \geqq 1,\left(T_{n, t}\right)_{t \geqq 0}$ satisfies the condition $\left(D^{*}\right)$, then so is $\left(T_{L, t}\right)_{t \geq 0}$. For each $f \in C_{K}^{+}(D)$, we choose an integer $n_{f} \geqq 1$ such that $f \in C_{K}^{+}\left(D_{n}\right)$ for all $n \geqq n_{f}$. Let $\left(f_{n, m}\right)_{m=1}^{\infty}$ be an associated family of $f$ with respect to $\left(T_{n, t}^{*}\right)_{t \geq 0}\left(n \geqq n_{f}\right)$. By Proposition 62 , we have

$$
V_{L, n}^{*} f \leqq V_{L, n+1}^{*} f \text { in } D \text { and } \lim _{n \rightarrow \infty} V_{L, n}^{*} f=V_{L}^{*} f \text { in } C(D)^{17)} .
$$

Hence we can choose inductively a sequence $\left(f_{n_{k}, m_{k}}\right)_{k=1}^{\infty}$ satisfying the following conditions (5.24), (5.25) and (5.26), where $n_{1} \geqq n_{f}$ and $n_{k}<n_{k+1}$ :

$$
V_{L}^{*} f-V_{L, n_{k}}^{*} f<\frac{1}{k} \text { on } \bar{D}_{n_{k-1}},
$$

17) We put $V_{L, n}^{*} f=0$ on $C D_{n}$. Then $V_{L, n}^{*} f \in C_{K}^{+}(D)$ by (5.21) and (5.22). 


$$
\begin{gathered}
V_{L, n_{k}}^{*} f_{n_{k}, m_{k}}<\frac{1}{k} \text { on } \bar{D}_{n_{k-1}}, \\
V_{L, n_{k-1}}^{*} f-V_{L, n_{k-1}}^{*} f_{n_{k-1}, m_{k-1}} \leqq V_{L, n_{k}}^{*} f-V_{L, n_{k}}^{*} f_{n_{k}, m_{k}} \text { in } D .
\end{gathered}
$$

We shall show that $\left(f_{n_{k}, m_{k}}\right)_{k=1}^{\infty}$ is an associated family of $f$ with respect to $\left(T_{L, t}^{*}\right)_{t \geqq 0}$. Since, for any $n \geqq n_{f}$ and any $m \geqq 1, L^{*} V_{L, n}^{*}\left(f-f_{n, m}\right)=-f+f_{n, m}$ in the sense of distributions in $D_{n}, f_{n, m} \in C_{K}^{+}\left(D_{n}\right)$, and hence we may assume that $f_{n, m} \in C_{K}^{+}(D)$. We have

$$
V_{L}^{*} f-V_{L}^{*} f_{n_{k}, m_{k}}=V_{L, n_{k}}^{*} f-V_{L, n_{k}}^{*} f_{n_{k}, m_{k}}(k \geqq 1),
$$

because $L^{*}\left(V_{L, n_{k}}^{*} f-V_{L, n_{k}}^{*} f_{n_{k}, m_{k}}\right)=f-f_{n_{k}, m_{k}}$ in the sense of distributions in $D$. This implies that $V_{L}^{*} f \geqq V_{L}^{*} f_{n_{k}, m_{k}}$ and $\operatorname{supp}\left(V_{L}^{*} f-V_{L}^{*} f_{n_{k}, m_{k}}\right)$ is compact. By (5.24), (5.25), (5.26) and (5.27), we have $V_{L}^{*} f_{n_{k-1}, m_{k-1}} \geqq V_{L}^{*} f_{n_{k}, m_{k}}$ in $D$ and $V_{L}^{*} f_{n_{k}, m_{k}} \leqq 2 / k$ on $\bar{D}_{n_{k-1} \text {. }}$. Thus we see that $\left(f_{n_{k}, m_{k}}\right)_{k=1}^{\infty}$ is an associated family of $f$ with respect to $\left(T_{L, t}^{*}\right)_{t \geqq 0}$. Consequently, it suffices to show that, for any subdomain $\Omega$ of $D$ satisfying the condition $(S),\left(T_{L, \Omega, t}\right)_{t \geqq 0}$ satisfies the condition $\left(D^{*}\right)$. For a fixed $y_{0} \in C \Omega$, we put $h(x)=G^{*}\left(x, y_{0}\right)$ for each $x \in \Omega$. Then $\inf _{x \in \Omega} h(x)>0, h \in C^{\infty}(\Omega)$ and $L^{*} h=0$ in $\Omega$. Let $f \in C_{K}^{+}(\Omega)$, and put $G_{\Omega}^{*}(x, y)=G_{\Omega}(y, x)$ and

$$
a=\min _{x \in \operatorname{supp}(f)} \frac{G_{a}^{*} f(x)}{h(x)}>0 .
$$

We choose a sequence $\left(\varphi_{n}\right)_{n=1}^{\infty} \subset C_{K}^{\infty}\left(R^{1}\right)$ such that, for each $n \geqq 1, \operatorname{supp}\left(\varphi_{n}\right)$ $\subset(a /(n+2), a /(n+1))$ and $\int \varphi_{n}(r) d r=1$. For any $0<r<a$, we put

$$
\Omega_{r}=\left\{x \in \Omega ; G_{\Omega}^{*} f(x)>\operatorname{rh}(x)\right\} .
$$

Then $\Omega_{r}$ is an open set with $\bar{\Omega}_{r} \subset \Omega$, because $G_{\Omega}^{*} f(x) \rightarrow 0$ as $x \rightarrow \partial \Omega$. Let $V_{L, \Omega}$ and $A_{L, \Omega}$ be the Hunt diffusion kernel for $\left(T_{L, Q, t}\right)_{t \geqq 0}$ and the infinitesimal generator of $\left(T_{L, \Omega, t}\right)_{t \geqq 0}$, respectively. Then, for any $V_{L, \Omega} \mu \in \mathscr{D}_{K}^{+}\left(A_{L, \Omega} ; \Omega_{r}\right)$,

$$
\int\left(G^{*} f-r h\right)^{+} d \mu=\int f d V_{L, \Omega} \mu-r \int G\left(y_{0}, x\right) d \mu(x)=\int f d V_{L, \Omega} \mu,
$$

because $\operatorname{supp}(\mu) \subset \Omega_{r}$. Hence Corollary 43 and (5.21) give that

$$
\left(G^{*} f-r h\right)^{+}(x)=\int f d\left(V_{L, \Omega} \varepsilon_{x}-V_{L, \Omega} \varepsilon_{x, C Q_{r}}^{\prime}\right)=G_{\Omega}^{*} f(x)-G_{\Omega}^{*} f_{C \Omega_{r}}^{\prime \prime}(x) \text { in } \Omega,
$$

where $\varepsilon_{x, C \Omega_{r}}^{\prime}$ is the $V_{L, \Omega}$-balayaged measure of $\varepsilon_{x}$ on $C \Omega_{r}$ and $f_{C \Omega_{r}}^{\prime \prime}$ is the $V_{L^{*}, \Omega}$-balayaged measure of $f d x$ on $C \Omega_{r}$. Put 


$$
g_{n}(x)=\int G^{*} f_{C \Omega_{r}}^{\prime \prime}(x) \varphi_{n}(r) d r(n=1,2, \cdots)
$$

Then we have

$$
g_{n}(x)=G_{\Omega}^{*} f(x)-h(x) \varphi_{n} * \psi\left(\frac{G^{*} f(x)}{h(x)}\right) \text { in } \Omega
$$

where $\psi(t)=t$ in $(0, \infty)$ and $\psi(t)=0$ in $(-\infty, 0]$. By $(5.32), g_{n} \in C^{\infty}\left(\Omega_{a / 2}\right)$ and, by (5.33), $g_{n} \in C^{\infty}(\Omega-\operatorname{supp}(f))$, i.e., $g_{n} \in C^{\infty}(\Omega) \quad(n=1,2, \cdots)$. By (5.32), $\left(g_{n}\right)_{n=1}^{\infty}$ converges decreasingly to 0 as $n \rightarrow \infty$. Since $\operatorname{supp}\left(G_{\Omega}^{*} f-g_{n}\right)$ $\subset \bar{\Omega}_{a /(n+1)}, G^{*} f-g_{n}$ is with compact support in $\Omega$. Since, for any $x \in \Omega$, the function $G_{\Omega}^{*} f_{C \Omega_{r}}^{\prime \prime}(x)$ of $r$ is finite continuous in $(0, a),(5.17)$ gives that $(0, a) \ni r \rightarrow f_{c \Omega_{r}}^{\prime \prime}$ is vaguely continuous, and hence $\int f_{c \Omega_{r}}^{\prime \prime} \varphi_{n}(r) d r$ is defined. Putting $f_{n}=-L^{*} g_{n}$, we see that $f_{n} \in C_{I S}^{+}(\Omega)$ and $f_{n}=\int f_{C \delta_{r}}^{\prime \prime} \varphi_{n}(r) d r$ in the sense of distributions. Thus $\left(f_{n}\right)_{n=1}^{\infty}$ is an associated family of $f$ with respect to $\left(T_{L, Q, t}^{*}\right)_{t \geq 0 \text {. }}$ This completes the proof.

In the usual way, we define the $L$-superharmonicity and the $L$-harmonicity.

Definition 71. A function $u$ in $D$ is said to be $L$-superharmonic (resp. $L$-harmonic) if $u$ satisfies the following three conditions:

(5.34) $u$ is lower semi-continuous (resp. continuous).

(5.35) $-\infty<u \leqq \infty, u \not \equiv \infty$ (resp. $-\infty<u<\infty$ ).

(5.36) $u$ is a locally integrable function in $D$ and $-L \mu \geqq 0$ (resp. $L u=0$ ) in the sense of distributions.

Similarly we define the $L^{*}$-superharmonicity and the $L^{*}$-harmonicity.

Proposition 72. Let $u$ be a lower semi-continuous function in $D$ satisfying $-\infty<u \leqq \infty$ and $u \neq \infty$. Then the following three conditions are equivalent:

(1) $u$ is L-superharmonic.

(2) If $\Omega$ is a relatively compact subdomain in $D$ and if $v$ is continuous on $\bar{\Omega}, L$-harmonic in $\Omega$ and satisfies $v(x) \leqq u(x)$ on $\partial \Omega$, then $v(x) \leqq u(x)$ in $\Omega$.

(3) For any relatively compact subdomain $\Omega$ in $D$ and any $x \in \Omega$, 


$$
u(x) \geqq \int u(y) d \varepsilon_{x, C \Omega}^{\prime \prime}(y),
$$

where $\varepsilon_{x, C \Omega}^{\prime \prime}$ is the $V_{L^{*}}$-balayaged measure of $\varepsilon_{x}$ on $C \Omega$.

Proof. The equivalence between (1) and (2) is shown by S. Itô (see, [12], Theorem 2).

(2) $\Rightarrow(3)$. Let $\left(\Omega_{n}\right)_{n=1}^{\infty}$ be a regular exhaustion of $\Omega$ such that $\Omega_{1} \ni x$. It is well-known that, for any $f \in C\left(\partial \Omega_{n}\right)$, the function $\int f d \varepsilon_{x, c \Omega_{n}}^{\prime \prime}$ of $x$ is $L$ harmonic in $\Omega_{n}$ (see, for example, [11]). In particular, if $f \leqq u$ on $\partial \Omega_{n}$, then (2) gives that $u(x) \geqq \int f d \varepsilon_{x, C \Omega_{n}}^{\prime \prime}$. By letting $f \uparrow u$ and $n \rightarrow \infty$, we obtain the required inequality.

The implication $(3) \sqcap(2)$ is directly followed from Proposition 42 and Corollary 43. This completes the proof.

Corollary 73. Let $u$ and $v$ be L-superharmonic functions in $D$. If $u=v d x$-a.e. in $D$, then $u=v$ everywhere.

Proof. First we remark that, for any $x \in D, G(x, x)=\infty$. Let $\Omega$ be a subdomain of $D$ satisfying the condition $(S)$. For a fixed $y \in C \Omega$, put $h(x)=G^{*}(x, y)$ on $\Omega$. For any $x_{0} \in \Omega$ and $r>0$, we denote by $\Omega_{r}$ the connected component of $\left\{x \in \Omega ; G^{*}\left(x_{0}, x\right)>r h(x)\right\}$ with $\Omega_{r} \ni x_{0}$ and choose $\varphi_{n} \in C_{K}^{\infty}\left(R^{1}\right)$ such that $\varphi_{n} \geqq 0, \int \varphi_{n}(r) d r=1$ and $\operatorname{supp}\left(\varphi_{n}\right) \subset(n, n+1)(n=$ $1,2, \cdots)$. Similarly as in Theorem $70, \int \varepsilon_{x_{0}, C \Omega_{r}}^{\prime \prime} \varphi_{n}(r) d r \in C_{K}^{\infty}(\Omega)$ in the sense of distributions, and hence

$$
\int\left(\int u d \varepsilon_{x_{0}, C \Omega_{r}}^{\prime \prime}\right) \varphi_{n}(r) d r=\int\left(\int v d \varepsilon_{x_{0}, C \Omega_{r}}^{\prime \prime}\right) \varphi_{n}(r) d r .
$$

Since $\left(\int \varepsilon_{x_{0}, C \Omega_{r}}^{\prime \prime} \varphi_{n}(r) d r\right)_{n=1}^{\infty}$ converges vaguely to $\varepsilon_{x_{0}}$ as $n \rightarrow \infty$, the lower semicontinuity of $u$, that of $v$ and (3) in Proposition 72 imply that $u\left(x_{0}\right)=v\left(x_{0}\right)$. The subdomain $\Omega$ and $x_{0}$ being arbitrary, we see Corollary 73 .

By the above corollary, we obtain the following

Proposition 74. Let $\mu \in M(D)$. If $\mu$ is $A_{L^{-}}$superharmonic (resp. $A_{L^{*}}$ superharmonic), then there exists one and only one L-superharmonic (resp. $L^{*}$-superharmonic) function $u$ in $D$ such that $\mu=u d x$.

Conversely, for an L-superharmonic (resp. $L^{*}$-superharmonic) function 


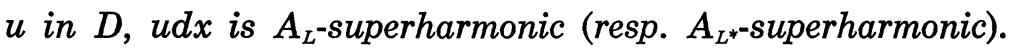

In order to prove Proposition 74, we use the following known lemma.

LEMMA 75 (see [18], p. 143). Let $\Omega$ be a domain in the $N$-dimensional Euclidean space $R^{N}(N \geqq 1)$ and $L$ be an elliptic differential operator of the analogous form to (5.1). If, for $\mu \in M(\Omega), L \mu \in C^{\infty}(\Omega)$ in the sense of distributions, then $\mu \in C^{\infty}(\Omega)$ in the sense of distributions. In particular, $L \mu=0$ in $\Omega$ implies $\mu \in C^{\infty}(\Omega)$ in the sense of distributions.

Proof of Proposition 74. Let $\mu \in M(D)$ be $A_{L}$-superharmonic. Then Remark 69 gives that $-L \mu \geqq 0$ in the sense of distributions. Let $\omega$ be a subdomain of $D$ satisfying the condition $(S)$ and $\lambda_{\omega}$ be the restriction of the positive measure $-L \mu$ to $\omega$. Put $\lambda=\mu-\left(G \lambda_{\omega}\right) d x$ in $\omega$. Then $L \lambda=0$ in $\omega$, and hence $\lambda=\varphi d x$ in $\omega$ by Lemma 75, where $\varphi \in C^{\infty}(\omega)$. The subdomain $\omega$ being arbitrary, we obtain that $\mu=u d x$, where $u$ is an $L$-superharmonic function in $D$. By Corollary 73, $u$ is uniquely determined. Let $u$ be an $L$-superharmonic function in $D$ and put $\mu=u d x$. Since $-L \mu \geqq 0$ in the sense of distributions in $D$, Remark 69 gives that $\mu$ is $A_{L}$-superharmonic if $\mu \in \mathscr{D}^{0}\left(A_{L}\right)$. Let $V_{L}^{*} f \in R_{K}\left(A_{L}\right)$. Then $\operatorname{supp}(f)$ is compact, and hence $\int|f| d \mu<\infty$, which implies $\mu \in \mathscr{D}^{0}\left(A_{L}\right)$. Thus $\mu$ is $A_{L}$-superharmonic. The rest of proof is similar. This completes the proof.

This implies evidently the following

Corollary 76. The infinitesimal generators $A_{L}$ and $A_{L^{*}}$ satisfy the condition $(\mathscr{L})$.

We denote by $S(L)$ the convex cone of all non-negative $L$-superharmonic functions in $D$ and by $H(L)$ the convex cone of all non-negative $L$-harmonic functions in $D$.

By Theorem 35, Corollary 73 and Proposition 74, we obtain the wellknown Riesz decomposition theorem.

Remark 77. For each $u \in S(L)$, there exists uniquely $(\nu, h) \in M^{+}(D) \times$ $H(L)$ such that $\mu=G \nu+h$.

Now we discuss the Martin compactification of $D$ for $L$.

Proposition 78. The Martin compactification $D^{*}$ of $D$ for $L$ is defined. Let $\mathfrak{S}_{1}$ be the essential part of the Martin boundary $\Gamma=D^{*}-D^{18)}$ and

18) $\widetilde{S}_{1}=\{\xi \in \Gamma$; the harmonic function $K(x, \xi)$ of $x$ is minimal $\}$. A positive harmonic function $u$ in $D$ is said to be minimal if, for any positive harmonic function $v$ in $D$, $v=c u$ with a positive constant $c$ whenever $u \geqq v$ in $D$. 
$K(x, \xi)$ be the Martin kernel on $D \times \Gamma$. If $h$ is positive L-harmonic in $D$, then there exists one and only one regular Borel positive measure $\mu$ on $\mathfrak{S}_{1}$ with $\int d \mu<\infty$ such that

$$
h(x)=\int_{\mathbb{S}_{1}} K(x, \xi) d \mu(\xi) \text { in } D .
$$

In the case of $c(x) \equiv 0$, the same assertion is obtained by S. Itô (see, [11], Theorem 5.3). Similarly we can prove Proposition 79 (see also [6], Chapter 11 and [18]).

For a constant $c>0$, we discuss non-negative solution of the following ideal boundary value problem:

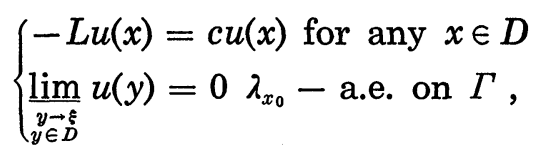

where $\lambda_{x_{0}}$ is the harmonic measure for a certain $x_{0} \in D$.

Denote by $E_{0}(L ; c)$ the set of non-negative functions of class $C^{\infty}$ in $D$ satisfying $(5.40)$ and by $E_{0}(L)=\bigcup_{c \geqq 0} E_{0}(L ; c)$.

Proposition 79. Let c be a non-negative constant. For each $\mu \in E_{0}\left(A_{L} ; c\right)$, there exists one and only one $u \in E_{0}(L ; c)$ such that $\mu=u d x$. Conversely, for any $u \in E_{0}(L ; c)$, we have $u d x \in E_{0}\left(A_{L} ; c\right)$.

Proof. Since $E_{0}\left(A_{L} ; 0\right)=\{0\}$ and $E_{0}(L ; 0)=\{0\}$, it suffices to show our conclusion in the case $c>0$. Let $\mu$ be a nonzero element of $E_{0}\left(A_{L} ; c\right)$. Then, by Propositions 45, 74, Corollary 73 and Remark 77, there exists one and only one $u \in S(L)$ such that $\mu=u d x$ and $u=c G u$. Since the function

$$
\int \underset{\substack{y \rightarrow \xi \\ y \in D}}{\lim _{y \rightarrow \xi}} u(y) \frac{K(x, \xi)}{K\left(x_{0}, \xi\right)} d \lambda_{x_{0}}(\xi)
$$

of $x$ is $L$-harmonic and $\leqq u$ in $D$, the second equality in (5.40) holds. Hence it suffices to show that $u \in C^{\infty}(D)$. We put inductively $G^{n+1}(x, y)=$ $\int G^{n}(x, z) G(z, y) d z$ and $G^{n} u(x)=\int G^{n}(x, y) u(y) d y$ for $n=1,2, \cdots$, where $G^{1}(x, y)=G(x, y)$. Then we have $u=c^{n} G^{n} u$. Let $\Omega$ be a relatively compact subdomain of $D$. When we consider $L$ as a differential operator in $\Omega, L$ is uniformly elliptic and all coefficients of $L$ are of class $C^{\infty}$ on $\bar{\Omega}$. 
Hence, for any $n \geqq N / 2+1, G_{\Omega}^{n}(x, y)$ is finite continuous in $\Omega \times \Omega$ (see, for example, [15], p. 1288), where the function $G_{\Omega}^{n}(x, y)$ is defined analogously to $G^{n}(x, y)$. Let $\Omega_{1}$ be another subdomain of $D$ such that $\bar{\Omega}_{1} \subset \Omega$ and $f$ be in $C_{K}^{+}(D)$ such that $0 \leqq f \leqq 1, f(x)=1$ on $\bar{\Omega}_{1}$ and $\operatorname{supp}(f) \subset \Omega$. Put $u_{1}=f u$ and $u_{2}=(1-f) u$. Then $G_{\Omega}^{n} u_{1}$ is finite continuous in $\Omega$ whenever $n \geqq$ $N / 2+1$. By remarking that, for any $k \geqq 1$,

$$
G^{k+1} u_{1}-G_{\Omega}^{k+1} u_{1}=G\left(G^{k} u_{1}-G_{\Omega}^{k} u_{1}\right)+G\left(G_{\Omega}^{k} u_{1}\right)-G_{\Omega}\left(G_{\Omega}^{k} u_{1}\right)
$$

and that, for any non-negative locally integrable function $g$ with $g \leqq u$, $G g-G_{\Omega} g$ is of class $C^{\infty}$ in $\Omega$ (see Lemma 75 and Corollary 73), we obtain inductively that $G^{n} u_{1}-G_{o}^{n} u_{1} \in C^{\infty}(\Omega)(n=1,2, \cdots)$. On the other hand, $G u_{2}$ is of class $C^{\infty}$ in $\Omega_{1}$ by Lemma 75. Let $\Omega_{2}$ be a subdomain of $D$ such that $\bar{\Omega}_{2} \subset \Omega_{1}$ and $\varphi$ be in $C_{R}^{\infty}(D)$ such that $0 \leqq \varphi \leqq 1, \varphi(x)=1$ on $\bar{\Omega}_{2}$ and $\operatorname{supp}(\varphi) \subset \Omega_{1}$. Then $G\left((1-\varphi) G u_{2}\right)$ is of class $C^{\infty}$ in $\Omega_{2}$ and $G\left(\varphi G u_{2}\right) \in C^{\infty}(D)$, because $\varphi G u_{2} \in C_{K}^{\infty}(D)$. The subdomain $\Omega_{2}$ being arbitrary, $G^{2} u_{2}$ is of class $C^{\infty}$ in $\Omega_{1}$. Inductively we see that, for any $n \geqq 1, G^{n} u_{2}$ is of class $C^{\infty}$ in $\Omega_{1}$. Thus $G^{n} u$ is finite continuous in $\Omega_{1}$ if $n \geqq N / 2+1$. The subdomain $\Omega$ and $\Omega_{1}$ being arbitrary, $u \in C(D)$. Since $u_{1} \in C_{K}^{+}(D), G_{\Omega}^{n} u_{1} \in C^{n}(\Omega)(n=$ $1,2, \cdots)$, and hence $G^{n} u_{1} \in C^{n}(\Omega)$. Consequently $G^{n} u \in C^{n}(\Omega)(n=1,2, \cdots)$, and so $u \in C^{\infty}(D)$.

Let $u \in E_{0}(L ; c)$. Then, by Remark $77, u=c G u+h$, where $h \in H(L)$. Since, for any $x \in D, \varliminf_{\substack{y \rightarrow \xi \\ y \in D}} u(y)=0 \lambda_{x}$-a.e. on $\Gamma$, the harmonic part $h$ of $u$ is equal to 0 , which implies that $u d x \in E_{0}\left(A_{L} ; c\right)$. This completes the proof.

Definition 80. A function $u$ in $D$ is said to be completely $L$-superharmonic in $D$ if, for any integer $n \geqq 0,(-L)^{n} u$ is $L$-superharmonic in $D$, where $(-L)^{0} u=u$ and $(-L)^{n} u$ is in the sense of distributions.

In particular, a completely $L$-superharmonic function $u$ in $D$ is said to be with zero conditions if $\underline{\lim }_{\substack{y \rightarrow x \\ y \in D}}(-L)^{n} u(y)=0$ for any $x \in \mathbb{S}_{1}$ and any $n=0,1, \cdots$.

We denote by $S C(L)$ the convex cone formed by all non-negative completely $L$-superharmonic functions in $D$ and by $S C_{0}(L)$ the convex cone formed by all non-negative completely $L$-superharmonic functions in $D$ with zero conditions.

Similarly as above, we see the following

Proposition 81. For each $\mu \in S C\left(A_{L}\right)$ (resp. $\in S C_{0}\left(A_{L}\right)$ ), there exists one 
and only one $u \in S C(L)$ (resp. $\in S C_{0}(L)$ ) such that $\mu=u d x$. Conversely, for any $u \in S C(L)$ (resp. $\in S C_{0}(L)$ ), $u d x \in S C\left(A_{L}\right)$ (resp. $\in S C_{0}\left(A_{L}\right)$ ).

Applying Theorem 53 to completely $L$-superharmonic functions, we obtain the following

TheOREM 82. We have $S C(L) \subset C^{\infty}(D)$ and the following assertions hold:

(1) If there exists an integer $k \geqq 1$ such that, for any $n$ with $1 \leqq n$ $\leqq k,\left(V_{L}\right)^{n}$ is defined as a diffusion kernel in $D$ and that $\left(V_{L}\right)^{k+1}$ is not defined, then, for each $u \in S C(L)$, there exists uniquely a finite family $\left(\lambda_{j}\right)_{j=0}^{k-1}$ of non-negative regular Borel measures on $\widetilde{\varsigma}_{1}$ with $\int d \lambda_{j}<\infty(j=0,1, \cdots$, $k-1)$ such that

$$
u(x)=\sum_{n=0}^{k-1} \int_{\mathfrak{S}_{1}} G^{n} \cdot K(x, \xi) d \lambda_{n}(\xi)
$$

where $G^{0} \cdot K(x, \xi)=K(x, \xi)$ and $G^{n} \cdot K(x, \xi)=\int G^{n}(x, y) K(y, \xi) d y$.

(2) If, for any integer $n \geqq 1,\left(V_{L}\right)^{n}$ is defined as a diffusion kernel on $D$, then, for each $u \in S C(L)$, there exist a sequence $\left(\lambda_{n}\right)_{n=0}^{\infty}$ of non-negative regular Borel measures on $\mathfrak{S}_{1}$ with $\int d \lambda_{n}<\infty(n=0,1, \cdots)$, a non-negative Borel measure $\sigma$ on $(0, \infty)$ with $\int d \sigma<\infty$ and a $\sigma$-measurable mapping $(0, \infty) \ni t \rightarrow u_{t} \in C^{\infty}(D)$ with $u_{t} \in E(L ; t)^{19)}$ such that, for any $y \in D$,

$$
u(y)=\sum_{n=0}^{\infty} \int_{\Theta_{1}} G^{n} \cdot K(y, \xi) d \lambda_{n}(\xi)+\int_{0}^{\infty} u_{t}(y) d \sigma(t) .
$$

Furthermore $\left(\lambda_{n}\right)_{n=0}^{\infty}$ is uniquely determined.

Proof. We first consider the case where the assumption of (1) holds. Let $u \in S C(L)$. Similarly as in Proposition 47, there exist uniquely a finite family $\left(h_{n}\right)_{n=0}^{k-1} \subset H(L)$ and $\nu \in \mathscr{D}^{+}\left(\left(V_{L}\right)^{k}\right)$ such that

$$
u d x=\sum_{n=0}^{k-1}\left(V_{L}\right)^{n}\left(h_{n} d x\right)+\left(V_{L}\right)^{k} \nu
$$

Since $\nu \in S\left(A_{L}\right)$, Theorem 35 gives that $\nu=V_{L}\left(-A_{L} \nu\right)+h_{k} d x$, where $h_{k} \in$ $H(L)$. Assume that $\nu \neq 0$. Let $\mu \in M_{K}^{+}(D)$ and $\Omega$ be a subdomain of $D$

19) We say that $t \rightarrow u_{t} \in C_{\infty}(D)$ is $\sigma$-measurable if, for any $x \in D$, the function $u_{t}(x)$ of $t$ is $\sigma$-measurable. 
satisfying the condition $(S)$ and $\operatorname{supp}(\mu) \subset \Omega$. We denote by $\mu_{C \Omega}^{\prime}$ the $V_{L^{-}}$ balayaged measure of $\mu$ on $C \Omega$. Then $V_{L} \mu-V_{L} \mu_{C \Omega}^{\prime} \in \mathscr{D}\left(\left(V_{L}\right)^{k}\right)$ and, by $\operatorname{supp}\left(\mu_{C \Omega}^{\prime}\right) \subset \partial \Omega$ and the domination principle for $V_{L}$, there exists a constant $c>0$ such that $V_{L} \mu_{C \Omega}^{\prime} \leqq c \nu$. Since $\nu \in \mathscr{D}\left(\left(V_{L}\right)^{k}\right), V_{L} \mu \in \mathscr{D}^{+}\left(\left(V_{L}\right)^{k}\right)$, and hence the mapping $M_{K}(D) \ni \mu \rightarrow\left(V_{L}\right)^{k}\left(V_{L} \mu\right) \in M(D)$ is defined and continuous, i.e., $\left(V_{L}\right)^{k+1}$ is defined as a diffusion kernel, which contradicts our assumption. This, Proposition 78 and (5.44) give (5.42), and (5.42) gives that $S C(L) \subset C^{\infty}(D)$.

Next we consider the case where the assumption of (2) holds. We remark that, for any $y \in D$, the mapping

$$
M^{+}(D) \supset\left\{v d x ; v \in E_{0}(L)\right\} \in v d x \rightarrow v(y) \in R^{+}
$$

is lower semi-continuous. This follows from the existence of a sequence $\left(f_{n}\right)_{n=1}^{\infty} \subset C_{K}^{+}(D)$ satisfying $\lim _{n \rightarrow \infty} f_{n} d x=\varepsilon_{y}$ (vaguely) and $v(y) \geqq \int v(z) f_{n}(z) d z$ for all $v \in S(L)$ (see the proof of Corollary 73). Let $u \in S C(L)$. By using Theorem 53, there exist a sequence $\left(h_{n}\right)_{n=0}^{\infty} \subset H(L)$, a non-negative Borel measure $\sigma$ on $(0, \infty)$ with $\int d \sigma<\infty$ and a bounded $\sigma$-measurable mapping $(0, \infty) \ni t \rightarrow u_{t} d x \in E_{0}\left(A_{L}\right)$ with $u_{t} \in E_{0}(L ; t)$ such that

$$
u d x=\sum_{n=0}^{\infty}\left(V_{L}\right)\left(h_{n} d x\right)+\int_{0}^{\infty}\left(u_{t} d x\right) d \sigma(t) .
$$

Hence Corollary 73 and (5.45) give that, for any $x \in D,(0, \infty) \ni t \rightarrow u_{t}(x)$ is $\sigma$-measurable and that

$$
u(x)=\sum_{n=0}^{\infty} G^{n} h_{n}(x)+\int_{0}^{\infty} u_{t}(x) d \sigma(t)
$$

This fact, Proposition 78 and the unicity of $\left(h_{n}\right)_{n=0}^{\infty}$ imply the assertion (2). It remains to show $S C(L) \subset C^{\infty}(D)$ under the assumption of (2). Let $n$ be an integer $\geqq N / 2+1$ and put $v_{n}=\int_{0}^{\infty} t^{n} u_{t} d \sigma(t)$. Then $(-L)^{n}\left(\int_{0}^{\infty} u_{t} d \sigma(t) d x\right)$ $=v_{n} d x$ in the sense of distributions in $D$, i.e., $v_{n}$ is locally integrable. Similarly as in Proposition 79, $G^{n} v_{n} \in C(D)$, and $\int_{0}^{\infty} u_{t} d \sigma(t)=G^{n} v_{n}$ (see corollary 73). In the same manner, $(-L)^{n} u \in C(D)$ in the sense of distributions for all $n \geqq 1$. This implies that $\int_{0}^{\infty} u_{t} d(t) \in C^{\infty}(D)$, and also, in the same manner as in Proposition 79, $\sum_{n=k}^{\infty} G^{n-k} h_{n}(x)$ is finite continuous in 
$D(k=0,1, \cdots), \sum_{n=0}^{\infty} G^{n} h_{n} \in C^{\infty}(D)$. This completes the proof.

M. V. Noviskiî [15] discusses completely $L$-superharmonic functions in the following setting. Let $D$ be a bounded domain in $R^{N}(N \geqq 2)$ of class $C^{1, \lambda}(\lambda>0)^{20)}$ and $L$ be a uniformly elliptic differential operator of the form

$$
L u(x)=\sum_{i, j=1}^{N} a_{i j}(x) \frac{\partial^{2} u}{\partial x_{i} \partial x_{j}}(x)+\sum_{i=1}^{N} b_{i}(x) \frac{\partial u}{\partial x_{i}}(x)+c(x) u(x)
$$

with coefficients $\in C^{\infty}(\bar{D})$, for $u \in C^{2}(D)$ and $x=\left(x_{1}, x_{2}, \cdots, x_{N}\right) \in D$, where $a_{i j}(x)=a_{j i}(x)$ and $c(x) \leqq 0$.

Evidently there exists the Green function $G(x, y)$ of $L$ on $D$ and we have $\lim _{\substack{x \rightarrow z \\ x \in D}} G(x, y)=\lim _{\substack{x \rightarrow z \\ x \in D}} G(y, x)=0$ for any $y \in D$ and any $z \in \partial D$.

Theorem 82 gives the main theorem of M. V. Noviskiľ's paper [15].

Corollary 83. Let $D$ be a bounded domain in $R^{N}(N \geqq 2)$ of class $C^{1, \lambda}(\lambda>0)$ and $L$ be given in (5.58). Denote by $\varphi_{1}$ a first eigen function $\geqq 0, \neq 0$ of $L$ with zero conditions on $\partial D$. A completely L-superharmonic function $u$ in $D^{21)}$ has the form

$$
u(x)=\sum_{k=0}^{\infty} \int_{\partial D}-\frac{\partial G^{k+1}}{\partial n_{y}}(x, y) d \mu_{k}(y)+c \varphi_{1}(x),
$$

where $\partial / \partial n_{y}$ denotes the outer normal derivative on $\partial D, \mu_{k}$ is a non-negative measure on $\partial D(k=0,1, \ldots)$ and $c$ is a non-negative constant. Furthermore $\left(\mu_{k}\right)_{k=0}^{\infty}$ and $c$ are uniquely determined.

LEMma 84 (see, [15], Lemma 3). Under the same conditions as above, a non-negative L-superharmonic function $f$ in $D$ is integrable if $f \in C^{2}(D)$.

Proof of Corollary 83. Similarly as in [11], §6, we may assume that the kernel $-\left(\partial / \partial n_{y}\right) G(x, y)$ on $D \times \partial D$ is the Martin kernel for $L$ and that $\partial D$ is the essential part of the Martin boundary. We remark that

$$
-\frac{\partial G^{k+1}}{\partial n_{y}}(x, y)=-\int G^{k}(x, z) \frac{\partial G}{\partial n_{y}}(z, y) d z \text { on } D \times \partial D(k=1,2, \cdots)
$$

20) The domain $D$ belongs to the class $C^{k, \lambda}(\lambda>0)$ if for an arbitrary $x_{0} \in \partial D$ there exists a neighborhood of $x_{0}$ in which $\partial D$ is specified by an equation $x_{i}=f\left(x_{1}, x_{2}, \cdots\right.$, $\left.x_{i-1}, x_{i+1}, \cdots, x_{N}\right)$, where $x=\left(x_{1}, x_{2}, \cdots, x_{N}\right) \in \partial D$ and $f$ is a $k$-times continuously differentiable function, the $k$-th derivatives of which satisfy a Hölder condition with exponent $\lambda>0$.

21) By Noviskir's definition, it is an infinitely differentiable function which satisfies the condition $(-L)^{n} u(x) \geqq 0, x \in D, n=0,1, \cdots$. 
and that there exists a first eigen function $\varphi_{1} \geqq 0, \neq 0$ of $L$ with zero conditions on $\partial D$ (see [13], Theorem 7.10). Hence it suffices to show that $E_{0}(L)=\left\{a \varphi_{1} ; a \in R^{+}\right\} . \quad$ Evidently $E_{0}(L) \ni \varphi$. By Proposition 79 and Lemma 84 , we have, for any $v \in E_{0}(L), \int v d x<\infty$, so that $G^{n} v$ is bounded if $n \geqq$ $N / 2+1$, i.e., $v$ is bounded, and hence $\lim _{\substack{y \rightarrow x \\ y \in D}} G v(y)=0$ for any $x \in \partial D$, i.e., $\lim _{\substack{y \rightarrow x \\ y \in D}} v(y)=0$ for any $x \in \partial D$. Thus we see that, for any $v \in E_{0}(L), \int v^{2} d x<\infty$. It is also known that there exists a first eigen function $\varphi_{1}^{*} \geqq 0, \neq 0$ of $L^{*}$ (see also [13], Theorem 7.10). Evidently $\int\left(\varphi_{1}^{*}\right)^{2} d x<\infty$. Let $c^{*}>0$ be the eigen value of $\varphi_{1}^{*}$. Then $\varphi_{1}^{*}=c^{*} G^{*} \varphi_{1}^{*}$. For any $v \neq 0 \in E_{0}(L)$, there exists $c>0$ such that $v=c G v$, which implies that $v>0$ on $D$. Since

$$
\int \varphi_{1}^{*} \cdot v d x=c^{*} \int G^{*} \varphi_{1}^{*} \cdot v d x=c^{*} \int \varphi_{1}^{*} \cdot G v d x=\frac{c^{*}}{c} \int \varphi_{1}^{*} \cdot v d x
$$

we have $c=c^{*}$, this implies that $E_{0}(L)=E_{0}\left(L ; c^{*}\right)$. Thus we see that, for any $v \in E_{0}(L)$ and any real number $t, \varphi_{1}-t v$ is also a first eigen function of $L$ with zero conditions on $\partial D$. By remarking that any first eigen function of $L$ with zero conditions on $D$ takes always non-negative values or non-positive values (see [13]), we obtain that, for any $v \in E_{0}(L) v=a \varphi_{1}$ with $a \in R^{+}$. This completes the proof.

\section{BIBLIOGRAPHY}

[1] C. Berg and G. Forst, Potential theory on locally compact abelian group, SpringerVerlag, Berlin, 1975.

[2] M. Brelot, Eléments de la théorie classique du potential, Les cours de Sorbonne, Paris, 1959.

[ 3 ] G. Choquet, Lectures on analysis, vol. 1, 2 and 3, W. A. Benjamin, 1969.

[4] G. Choquet and J. Deny, Aspects linéaires de la théorie du potentiel. Théorème de dualité et applications, C. R. Acad. Sci. Paris, 243 (1959), 764-767.

[5] G. Choquet and J. Deny, Sur l'équation de convolution $\mu=\mu * \sigma$, C. R. Acad. Sci. Paris, 250 (1960), 799-801.

[6] C. Constantinescue and A. Cornea, Potential theory on harmonic space, SpringerVerlag, Berlin, 1972.

[ 7 ] J. Deny, Noyaux de convolution de Hunt et noyaux associés à une famille fondamentale, Ann. Inst. Fourier, Grenoble, 12 (1962), 643-667.

[8] M. Itô, Sur le principle relatif de domination pour les noyaux de convolution, Hiroshima Math. J., 5 (1975), 293-350.

[9] S. Itô, Fundamental solution of parabolic differential equations and boundary value problems, Japan. J. Math., 27 (1957), 55-102.

[10] S. Itô, On existence of Green function and positive superharmonic functions for 
linear elliptic operators of second order, J. Math. Soc. Japan 16 (1964), 299-306.

[11] S. Itô, Martin boundary for linear elliptic differential operators of second order in a manifold, J. Math. Soc. Japan, 16 (1964), 307-334.

[12] S. Itô, On definitions of superharmonic functions, Ann. Inst. Fourier, Grenoble, 25 (1975), 309-316.

[13] M. A. Krasnosel'skii, Positive solution of operator equations, P. Noodfoff Ltd. Groningen, The Netherlands, 1960.

[14] C. Miranda, Partial differential equations of elliptic type, Springer-Verlag, Berlin, 1970.

[15] M. V. Noviskiì, Representation of completely $L$-superharmonic functions, Math. Nauk. Izv., 9 (1975), no. 6, 1279-1296.

[16] M. V. Noviskiì, Integral representation of totally excessive elements, Soviet Math. Dokl., 16 (1975), no. 6, 1510-1514.

[17] R. R. Phelps, Lecture on Choquet's theorem, Van Nostrand, Princeton, N. J., 1966.

[18] L. Schwarts, Théorie des distributions, Hermann, Paris, 1966.

[19] M. G. Šur, The Martin boundary for a linear elliptic second-order operator, Izv. Akad Nauk. SSSR, ser Math., 27 (1963), 45-60.

\author{
Department of Mathematics \\ Faculty of Science \\ Nagoya University \\ Nagoya, Japan
}

